This item was submitted to Loughborough's Research Repository by the author.

Items in Figshare are protected by copyright, with all rights reserved, unless otherwise indicated.

\title{
Nonlinear geostrophic adjustment in the presence of a boundary
}

PLEASE CITE THE PUBLISHED VERSION

LICENCE

CC BY-NC-ND 4.0

\section{REPOSITORY RECORD}

Reznik, G.M., and Roger H.J. Grimshaw. 2019. "Nonlinear Geostrophic Adjustment in the Presence of a Boundary". figshare. https://hdl.handle.net/2134/710. 


\title{
NONLINEAR GEOSTROPHIC ADJUSTMENT IN THE PRESENCE OF A BOUNDARY
}

\author{
G.M. Reznik \\ P.P. Shirshov Institute of Oceanology, Moscow, Russia
}

R. Grimshaw

Department of Mathematical Sciences, Loughborough University, UK 


\begin{abstract}
The process of nonlinear geostrophic adjustment in the presence of a boundary (i.e. in a half-plane bounded by a rigid wall) is examined in the framework of a rotating shallow water model, using an asymptotic multiple time-scale theory based on the assumed smallness of the Rossby number $\varepsilon$. The spatial scale is of the order of the Rossby scale. Different initial states are considered: periodic, “step"-like, and localised. In all cases the initial perturbation is split in a unique way into slow and fast components evolving with characteristic time-scales $f^{-1}$ and $(\varepsilon f)^{-1}$, respectively. The slow component is not influenced by the fast one, at least for times $t \leq(\varepsilon f)^{-1}$, and remains close to geostrophic balance. The fast component consists mainly of linear inertia-gravity waves rapidly propagating outward from the initial disturbance, and Kelvin waves confined near the boundary.

The theory provides simple formulae allowing us to construct the initial profile of the Kelvin wave, given arbitrary initial conditions. With increasing time, the Kelvin wave profile gradually distorts due to nonlinear wave self-interaction, the distortion being described by the equation of a simple wave. The presence of Kelvin waves does not prevent the fast-slow splitting, in spite of the fact that the frequency gap between the Kelvin waves and slow motion is absent. The possibility of such splitting is explained by the special structure of the Kelvin waves in each considered case.

The slow motion on times $\quad t \leq(\varepsilon f)^{-1}$ is governed by the well-known quasigeostrophic potential vorticity equation for the elevation. The theory provides an algorithm to determine initial slow and fast fields, and the boundary conditions to any order in $\varepsilon$. For the periodic and step-like initial conditions, the slow component behaves in the "usual" way conserving mass, energy, and enstrophy. In the case of a localised initial disturbance the total mass of the lowest-order slow component is not conserved, and conservation of the total mass is provided by the first-order slow correction and the Kelvin wave.

On longer times $t \leq\left(\varepsilon^{2} f\right)^{-1}$ the slow motion obeys the so-called modified quasigeostrophic potential vorticity equation. The theory provides initial and boundary conditions for this equation. This modified equation exactly coincides with the "improved" quasigeostrophic potential vorticity equation, derived by Reznik, Zeitlin \& Ben Jelloul (2001), in the "step"-like and localised cases. In the periodic case this equation contains an additional term due to the Kelvin wave self-interaction, this term depending on the initial Kelvin wave profile.
\end{abstract}




\section{Introduction}

Geostrophic adjustment in a rotating fluid is the tendency for the large-scale part of the initial perturbation to reach a state of geostrophic equilibrium; at the same time the remaining part, consisting of rapidly propagating inertia-gravity (IG) waves gradually decays at a fixed space point with increasing time. The study of this process (playing a very important role in atmospheric and oceanic dynamics) started in the pioneering work by Rossby (1938). A thorough linear analysis of geostrophic adjustment has been given in a number of well-known works (e.g. Obukhov 1949, Monin \& Obukhov 1958, Gill 1976). The lowest-order nonlinear corrections were discussed in the review by Blumen (1972) (where an extensive bibliography of the early works can be found) and more recently by Dewar \& Killworth (1995).

The problem of nonlinear geostrophic adjustment is strongly related to the problem of balanced models which are used to describe the slow (balanced) part of the motion (e.g. Warn et al, 1995, Medvedev, 1997). The fundamental question is about the possibility to split an arbitrary motion into a slow (balanced) and a fast component in such a way that the slow component is not influenced by the fast one for long enough times. Note that the splitting is a more general concept than adjustment, since splitting is possible when the fast component consists of "non-radiating" oscillations and permanently co-exist with the slow component, as in the cases of periodic motion or frontal dynamics. Periodic (in both horizontal directions) motion was examined by Embid \& Majda (1996), Babin, Mahalov \& Nikolaenko (1998a,b) in the framework of a barotropic rotating shallow water (RSW) model when the relative elevation and the Rossby number $\varepsilon$ are small. It was shown that the resulting field is split in a unique way into slow and fast components evolving with characteristic time scales $f^{-1}$ and $(\varepsilon f)^{-1}$, respectively, where $f$ is the Coriolis parameter. The slow component is not influenced by the fast one and remains close to geostrophic balance, being governed by the well-known quasi-geostrophic (QG) dynamics. The fast component is a superposition of IG waves with amplitudes slowly changing in time due to nonlinear interactions between the waves and slow motion (serving here as a catalyst).

These results were generalised by Reznik, Zeitlin \& Ben Jelloul (2001) (hereafter referred to as RZB) to the case of an arbitrary localized perturbation on an unbounded plane. The scenario of adjustment depends on the characteristic scale and/or initial relative elevation of the free surface. For small relative elevations the slow motion obeys well-known QG equation for times $t \leq(f \varepsilon)^{-1}$ while modifications to this equation for longer times $t \leq\left(f \varepsilon^{2}\right)^{-1}$ is found. The fast component consists mainly of linear IG waves rapidly propagating outward from the initial perturbation; the 
nonlinear interaction between these waves and slow component is of no importance, contrary to the periodic case. For large relative elevations (the frontal dynamics regime) the slow field is governed by the frontal geostrophic dynamics equation. The fast component in this case is a spatially localised packet of inertial oscillations evolving on the background of the slow component of the flow and experiencing slow modulations obeying a Schrodinger-type equation with coefficients depending on the slow motion.

The physical reasons of the slow-fast splitting are the Lagrangian conservation of potential vorticity, the fact that IG waves do not carry the potential vorticity, and the gap in the spectrum of small perturbation in RSW due to rotation which, in particular, blocks the Lighthill radiation of IG waves.

The question addressed in this paper is how these results are modified in the presence of a horizontal boundary. Obviously, this question is of special interest for oceanographers. An essential new factor absent in unbounded fluid is the presence of Kelvin waves propagating along the boundaries in the rotating fluid. Gill (1976) examined the linear geostrophic adjustment in a channel and revealed that the Kelvin waves play a key role in establishing of the boundary conditions for the resulting geostrophic mode. As for the nonlinear adjustment, the Kelvin waves can be of even more importance than for the linear one, since there is no spectral gap between the slow mode and Kelvin waves: these waves can possess arbitrarily small frequencies. This means that Lighthill radiation of Kelvin waves is possible, at least, in principle.

The aim of this paper is to investigate the influence of boundaries on the process of nonlinear geostrophic adjustment. For simplicity we use again the barotropic RSW model and consider the motion on a half-plane bounded by a rigid wall. The Rossby number is assumed to be small and the asymptotic multiple time-scale procedure developed in RZB is applied for analysis.

The paper is organised as follows. In Section 2 the model is formulated. In Section 3 we examine the lowest-order solution for various initial conditions; the solution obtained describes the linear geostrophic adjustment of an arbitrary initial field on the half-plane. Nonlinear dynamics of the lowest-order slow motion is analysed in Section 4. The first-order solution is discussed in Section 5; we demonstrate that slow-fast splitting is possible at higher orders in Rossby number, at least up to terms $O\left(\varepsilon^{2}\right)$. A modified quasigeostrophic potential vorticity equation describing the slow component on times longer than typical geostrophic time-scale, for $t \leq\left(f \varepsilon^{2}\right)^{-1}$, is derived in Section 6. A summary of our results is presented in Section 7. The asymptotic behaviour of the lowest- and first-order fields is considered in Appendixes A and B. 


\section{Model}

The RSW model consists of the horizontal momentum and mass conservation equations

for the thin free-surface layer under the influence of the Coriolis force and gravity on the rotating plane $(x, y)$ :

$$
\begin{aligned}
& \frac{\partial u}{\partial t}+\varepsilon\left(u \frac{\partial u}{\partial x}+v \frac{\partial u}{\partial y}\right)-v=-\frac{\partial h}{\partial x} \\
& \frac{\partial v}{\partial t}+\varepsilon\left(u \frac{\partial v}{\partial x}+v \frac{\partial v}{\partial y}\right)+u=-\frac{\partial h}{\partial y} \\
& \frac{\partial h}{\partial t}+(1+\varepsilon h)\left(\frac{\partial u}{\partial x}+\frac{\partial v}{\partial y}\right)+\varepsilon\left(u \frac{\partial h}{\partial x}+v \frac{\partial h}{\partial y}\right)=0
\end{aligned}
$$

where $\mathbf{v}=(u(x, y, t), v(x, y, t))$ is the two-dimensional velocity field and $H=H_{0}(1+\varepsilon h(x, y, t))$ is the free surface elevation with the rest state corresponding to the constant $H_{0}$. Equations (2.1) are written in non-dimensional form, the Rossby scale $R_{d}=\sqrt{g H_{0}} / f$ and the reciprocal Coriolis parameter $f^{-1}$ being chosen as the space and the time scales, respectively; $g$ is the gravity acceleration, the parameter $\varepsilon=U / f R_{d}$ is the Rossby number, and $U$ is the horizontal velocity scale.

The fields $u, v, h$ are known at the initial moment,

$u=u_{I}(x, y), \quad v=v_{I}(x, y), \quad h=h_{I}(x, y)$ at $t=0$,

and obey the no flux boundary condition on the rigid wall $y=0$,

$v=0, \quad y=0$.

The initial fields (2.2) are not entirely arbitrary and satisfy the following conditions at the wall $y=$ 0 ,

$v_{I}=0, \quad u_{I}=-\frac{\partial h_{I}}{\partial y}, \quad y=0$,

to be consistent with the equations (2.1b), (2.3).

The vorticity equation follows from (2.1), 
$\frac{\partial(\varsigma-h)}{\partial t}+\varepsilon\left\{\frac{\partial[u(\varsigma-h)]}{\partial x}+\frac{\partial[v(\varsigma-h)]}{\partial y}\right\}=0$,

$\varsigma=\frac{\partial v}{\partial x}-\frac{\partial u}{\partial y}$

It is seen from $(2.1),(2.5)$ that the fields $u, v, h$ on the one hand and the vorticity field $\varsigma-h$ from the other hand, are characterised, generally, by different time scales. The typical time of $u, v, h$ is equal to the inertial time $T_{i}=f^{-1}$ and $\varsigma-h$ changes in time at the advective time scale $T_{a}=R_{d} / U$. The Rossby number $\varepsilon$ is the ratio between these scales,

$$
\frac{T_{i}}{T_{a}}=\frac{U}{f R_{d}}=\varepsilon
$$

In what follows we assume the advective time scale to be much larger than the inertial one which means that the Rossby number is small,

$$
\varepsilon<1 \text {. }
$$

Both the fast changes due to IG waves activity and and the slow changes of the vorticity $\varsigma-h$ are present in the evolution of the initial field (2.2) and the problem of nonlinear adjustment is to determine their mutual influence. The geostrophic adjustment in the bounded half-plane is schematically represented in Fig. 1.

Three types of the initial conditions $\left(u_{I}, v_{I}, h_{I}\right)$ will be considered:

1. Periodic (in $x$ ) boundary conditions,

$$
\left(u_{I}, v_{I}, h_{I}\right)=\sum_{m=-\infty}^{m=\infty}\left(u_{I m}, v_{I m}, h_{I m}\right) e^{i m x} ;
$$

where the functions $u_{I m}, v_{I m}, h_{I m}$ depend on $y$ only.

2. A zonal "step",

$$
\left(u_{I}, v_{I}, h_{I}\right) \rightarrow\left(u_{I}^{( \pm)}(y), 0, h_{I}^{( \pm)}(y)\right), \quad x \rightarrow \pm \infty .
$$

3. Localized motion,

$$
\left(u_{I}, v_{I}, h_{I}\right) \rightarrow 0, \quad x \rightarrow \pm \infty .
$$

In all cases the initial motion is assumed to be localized in the $y$-direction. At first formulae not depending on the type of initial conditions will be derived, and then the special cases $(2.9 \mathrm{a}, \mathrm{b}, \mathrm{c})$ are discussed.

Following RZB the solution to the system (2.1), (2.2), (2.3) is sought in the form of the following asymptotic expansions:

$$
\begin{aligned}
& u=u_{0}\left(x, y, t, T_{1}, \ldots\right)+\varepsilon u_{1}\left(x, y, t, T_{1}, \ldots\right)+\ldots \\
& v=v_{0}\left(x, y, t, T_{1}, \ldots\right)+\varepsilon v_{1}\left(x, y, t, T_{1}, \ldots\right)+\ldots \\
& h=h_{0}\left(x, y, t, T_{1}, \ldots\right)+\varepsilon h_{1}\left(x, y, t, T_{1}, \ldots\right)+\ldots
\end{aligned}
$$


Here $T_{n}=\varepsilon^{n} t, n=1,2 \ldots$ are the slow times needed to prevent the solution from a secular growth in time.

\section{Lowest-order solution (linear adjustment)}

Splitting

It is of convenience to use the vorticity equation (2.5) instead of the mass conservation equation (2.1c) in the sequel. Substitution of (2.10) into (2.1a,b), (2.2), (2.3), and (2.5) gives in the lowest order:

$$
\begin{aligned}
& \frac{\partial u_{0}}{\partial t}-v_{0}=-\frac{\partial h_{0}}{\partial x}, \quad \frac{\partial v_{0}}{\partial t}+u_{0}=-\frac{\partial h_{0}}{\partial y}, \quad \frac{\partial\left(\varsigma_{0}-h_{0}\right)}{\partial t}=0, \\
& \left.v_{0}\right|_{y=0}=0, \quad\left(u_{0}, v_{0}, h_{0}\right)_{t=0}=\left(u_{I}, v_{I}, h_{I}\right) .
\end{aligned}
$$

By virtue of (3.1c) the lowest-order vorticity does not depend on the fast time $t$, i.e.

$\varsigma_{0}-h_{0}=\Pi_{0}\left(x, y, T_{1}, \ldots\right)$.

The solution to (3.1) is represented as a sum of fast and slow components,

$\left(u_{0}, v_{0}, h_{0}\right)=\left(\tilde{u}_{0}, \tilde{v}_{0}, \tilde{h}_{0}\right)\left(x, y, t, T_{1}, \ldots\right)+\left(\bar{u}_{0}, \bar{v}_{0}, \bar{h}_{0}\right)\left(x, y, T_{1}, \ldots\right)$,

the fast component having zero average with respect to the fast time $t$, i.e.

$\left\langle\tilde{u}_{0}\right\rangle_{t}=\left\langle\widetilde{v}_{0}\right\rangle_{t}=\left\langle\tilde{h}_{0}\right\rangle_{t}=0$

Here the averaging is defined as follows

$$
\langle a\rangle_{t}=\lim \frac{1}{T_{a v}} \int_{0}^{T_{a v}} a d t, \quad \text { as } T_{a v} \rightarrow \infty .
$$

Applying the averaging (3.5) to $(3.1 \mathrm{a}, \mathrm{b}),(3.2)$ we obtain the following equations for the slow and fast components:

$$
\begin{aligned}
& \bar{v}_{0}=\frac{\partial \bar{h}_{0}}{\partial x}, \quad \bar{u}_{0}=-\frac{\partial \bar{h}_{0}}{\partial y}, \quad \bar{\zeta}_{0}-\bar{h}_{0}=\nabla^{2} \bar{h}_{0}-\bar{h}_{0}=\Pi_{0}, \\
& \left.\bar{v}_{0}\right|_{y=0}=0 ; \\
& \frac{\partial \tilde{u}_{0}}{\partial t}-\tilde{v}_{0}=-\frac{\partial \tilde{h}_{0}}{\partial x}, \quad \frac{\partial \tilde{v}_{0}}{\partial t}+\tilde{u}_{0}=-\frac{\partial \tilde{h}_{0}}{\partial y}, \quad \tilde{\zeta}_{0}-\tilde{h}_{0}=0, \\
& \left.\tilde{v}_{0}\right|_{y=0}=0 .
\end{aligned}
$$




\section{Initial conditions for fast and slow zonal velocities}

Of course, the splitting (3.3) is incomplete unless the initial conditions for each component are determined. This can be easily done for the meridional velocity $v_{0}$. The initial field $\bar{v}_{0}$ (in what follows the initial fields are designated by the subscript " $P$ ") for the slow meridional velocity satisfies the equation readily deriving from the vorticity equation (3.6c) and the geostrophic equation (3.6a):

$\nabla^{2} \bar{v}_{0 I}-\bar{v}_{0 I}=\frac{\partial \Pi_{I}}{\partial x}, \quad \Pi_{I}=\varsigma_{I}-h_{I}=\frac{\partial v_{I}}{\partial x}-\frac{\partial u_{I}}{\partial y}-h_{I}$

Equation (3.8a) should be solved under the boundary condition

$\left.\bar{v}_{0 I}\right|_{y=0}=0$.

System (3.8) allows us to find the initial field $\bar{v}_{0 I}$. However, the initial field $\bar{h}_{0 I}$ cannot be determined at this stage since (3.8c), (3.6a) imply only that $\bar{h}_{0 I}$ is a constant at $y=0$,

$\left.\bar{h}_{0 I}\right|_{y=0}=\bar{h}_{0 I}^{(B)}=$ constant,

and one has to determine this constant $\bar{h}_{0 I}^{(B)}$, to make the problem for $\bar{h}_{0 I}$ complete. Finding this constant is related to analysis of the Kelvin waves arising when the initial conditions (2.2) are not geostrophically balanced.

Knowing $\bar{\nabla}_{0}$ from (3.8) one can find the "fast" initial field

$\tilde{v}_{0 I}=v_{I}-\bar{v}_{0 I}=F(x, y)$.

Another initial condition for $\widetilde{v}_{0}$ follows from (3.6b), (3.7b), and (3.1e):

$\left.\frac{\partial \tilde{v}_{0}}{\partial t}\right|_{t=0}=-\left(u_{I}+\frac{\partial h_{I}}{\partial y}\right)=G(x, y)$.

The equation for $\widetilde{v}_{0}$ is derived from $(3.7 \mathrm{a}, \mathrm{b}, \mathrm{c})$ (see e.g. RZB for details):

$L_{w} \tilde{v}_{0}=0, \quad L_{w}=-\frac{\partial^{2}}{\partial t^{2}}+\nabla^{2}-1$.

This equation together with the initial conditions (3.10), and the boundary condition (3.7d) allows us to determine the field $\widetilde{v}_{0}$ given initial conditions (2.2). 
It is convenient to construct the solution for $\widetilde{V}_{0}$ extended to the whole plane instead of the half-plane. To do that we determine the odd functions

$$
\left(F^{o d}, G^{o d}\right)=\left\{\begin{array}{c}
(F(x, y), G(x, y)), \quad y>0 \\
-(F(x,-y), G(x,-y)), \quad y<0
\end{array}\right.
$$

and search for the solution to equation (3.11) which is bounded at infinity, and valid on the whole plane, with the initial conditions

$$
\tilde{v}_{0 I}=F^{o d}(x, y),\left.\quad \frac{\partial \tilde{v}_{0}}{\partial t}\right|_{t=0}=G^{o d}(x, y) \text {. }
$$

The resulting field $\widetilde{\nabla}_{0}$ is also odd and therefore satisfies the boundary condition $(3.7 \mathrm{~d})$. The linear problem (3.11), (3.13) is conveniently solved using Fourier series (in the periodic case (2.9a)), and Fourier integrals. The resulting solutions in all cases are represented as a superposition of harmonic IG waves with constant amplitudes. In Appendix A the formulae for the solution to (3.11), (3.13) are presented for all cases $(2.9 \mathrm{a}, \mathrm{b}, \mathrm{c})$.

An important point is that the problem (3.11), (3.13) determines only the fast time dependence of $\widetilde{v}_{0}$ and to introduce the slow time dependence we represent $\tilde{v}_{0}$ as a sum (cf. RZB) $\tilde{v}_{0}=\tilde{v}_{0 f}(x, y, t)+\tilde{v}_{00}\left(x, y, t, T_{1}, T_{2}, \ldots\right)$.

Here $\widetilde{v}_{O f}$ is the solution to the problem (3.11), (3.13) and $\widetilde{v}_{O C}$ has a form analogous to $\widetilde{v}_{O f}$ (i.e. it is also is a superposition of linear IG waves which is an odd function of $y$ ) but with the still unknown wave amplitudes depending on the slow times (see Appendix A and RZB for more details). Obviously, $\tilde{v}_{00}$ is a solution to the homogeneous wave equation (3.11) and the only condition to be satisfied by $\tilde{v}_{00}$ is that it is zero at $T_{1}=T_{2}=\ldots 0$. Due to this condition the correction $\tilde{v}_{00}$ can be neglected for times $t<<\varepsilon^{-1}$. This additional term in the lowest-order wave solution and analogous terms in other fields are, in principle, necessary to avoid secular growth at higher order of the perturbation theory.

To find the fields $\widetilde{u}_{0}, \widetilde{h}_{0}$ we use the equations following from $(3.7 \mathrm{a}, \mathrm{b}, \mathrm{c})$ :

$$
\frac{\partial^{2} \tilde{h}_{0}}{\partial y^{2}}-\tilde{h}_{0}=-\left(\frac{\partial \tilde{v}_{0}}{\partial x}+\frac{\partial^{2} \tilde{v}_{0}}{\partial t \partial y}\right), \quad \frac{\partial^{2} \tilde{u}_{0}}{\partial y^{2}}-\tilde{u}_{0}=\frac{\partial \tilde{v}_{0}}{\partial t}+\frac{\partial^{2} \tilde{v}_{0}}{\partial x \partial y}
$$

The solutions to $(3.15 \mathrm{a}, \mathrm{b})$ are conveniently written in the form 
$\widetilde{u}_{0}=\widetilde{u}_{01}\left(x, y, t, T_{1}, \ldots\right)+C_{u}^{(0)}\left(x, t, T_{1}, \ldots\right) e^{-y}$,

$\widetilde{h}_{0}=\widetilde{h}_{01}\left(x, y, t, T_{1}, \ldots\right)+C_{h}^{(0)}\left(x, t, T_{1}, \ldots\right) e^{-y}$,

where $C_{u}^{(0)}$ and $C_{h}^{(0)}$ are some arbitrary functions which have to be determined and $\tilde{u}_{01}, \tilde{h}_{01}$ are expressed in terms of $\widetilde{v}_{0}$ :

$\tilde{u}_{01}=-\frac{1}{2}\left(\frac{\partial s^{+}}{\partial t}+\frac{\partial s^{-}}{\partial x}\right), \quad \tilde{h}_{01}=\frac{1}{2}\left(\frac{\partial s^{+}}{\partial x}+\frac{\partial s^{-}}{\partial t}\right)$

$s^{ \pm}=e^{y} \int_{y}^{\infty} \tilde{v}_{0} e^{-y} d y \pm e^{-y} \int_{-\infty}^{y} \tilde{v}_{0} e^{y} d y$.

The slow time dependence in $\widetilde{u}_{01}, \widetilde{h}_{01}$ is due to the term $\widetilde{v}_{0 C}$ in (3.14).

\section{The Kelvin wave and the initial slow field $\bar{h}_{0}$}

To determine the functions $C_{u}^{(0)}, C_{h}^{(0)}$ we write equations $(3.7 \mathrm{a}, \mathrm{b}, \mathrm{c})$ at $y=0$ :

$\frac{\partial \tilde{u}_{0}}{\partial t}+\frac{\partial \tilde{h}_{0}}{\partial x}=0, \quad \tilde{u}_{0}=-\frac{\partial \tilde{h}_{0}}{\partial y}, \quad \tilde{h}_{0}=-\frac{\partial \tilde{u}_{0}}{\partial y}, \quad y=0$.

Using the representation (3.17) and the fact that $\widetilde{v}_{0}$ is an odd function of $y$, one can show that

$\left.\tilde{u}_{0}\right|_{y=0}=-\int_{0}^{\infty} \frac{\partial \tilde{v}_{0}}{\partial x} e^{-y} d y+C_{u}^{(0)},\left.\quad \frac{\partial \tilde{u}_{0}}{\partial y}\right|_{y=0}=-\int_{0}^{\infty} \frac{\partial \tilde{v}_{0}}{\partial t} e^{-y} d y-C_{u}^{(0)} ;$

$\left.\tilde{h}_{0}\right|_{y=0}=\int_{0}^{\infty} \frac{\partial \tilde{v}_{0}}{\partial t} e^{-y} d y+C_{h}^{(0)},\left.\quad \frac{\partial \tilde{h}_{0}}{\partial y}\right|_{y=0}=\int_{0}^{\infty} \frac{\partial \tilde{v}_{0}}{\partial x} e^{-y} d y-C_{h}^{(0)}$.

Substituting (3.19a), (3.20b) into (3.18b) we obtain that

$$
C_{u}^{(0)}=C_{h}^{(0)}
$$

In turn, the substitution of (3.19a), (3.20a) into (3.18a) and the taking into account of (3.21), gives the equation for $C_{u}^{(0)}$ :

$\frac{\partial C_{u}^{(0)}}{\partial t}+\frac{\partial C_{u}^{(0)}}{\partial x}=0$

whence

$C_{u}^{(0)}=C_{h}^{(0)}=K_{w}^{(0)}\left(x-t, T_{1}, \ldots\right)$ 
Thus the last terms in $(3.16 \mathrm{a}, \mathrm{b})$ describe the Kelvin wave propagating in such a way that the boundary is to the right of the propagation direction.

To determine the Kelvin wave profile we consider equation (3.20a) at the initial moment and use (3.10); as a result we have:

$\left.\tilde{h}_{0 I}\right|_{y=0}=-\int_{0}^{\infty}\left(u_{I}+\frac{\partial h_{I}}{\partial y}\right) e^{-y} d y+K_{w}^{(0)}(x)$.

Bearing in mind that

$$
\tilde{h}_{0 l}+\bar{h}_{\mathrm{Ol}}=h_{\mathrm{l}}
$$

we obtain the equation (see (3.9)):

$$
K_{w}^{(0)}(x)=\int_{0}^{\infty}\left(u_{I}+\frac{\partial h_{I}}{\partial y}\right) e^{-y} d y+\left.h_{I}\right|_{y=0}-\left.\bar{h}_{0 I}\right|_{y=0},
$$

relating the Kelvin wave profile to the constant boundary value $\bar{h}_{0 I}^{(B)}(3.9)$ of the initial slow elevation $\bar{h}_{0 I}$ which is also unknown. To calculate this constant and the Kelvin wave profile we use condition (3.4). As we show in Appendix B, the fields $\tilde{v}_{0}, \tilde{u}_{01}, \tilde{h}_{01}$ decay with increasing time $t$ so that

$$
\tilde{v}_{0}=O\left(\frac{1}{t^{3 / 2}}\right), \quad \tilde{u}_{01}=O\left(\frac{1}{t^{3 / 2}}\right), \quad \tilde{h}_{01}=O\left(\frac{1}{t^{3 / 2}}\right), \quad t \rightarrow \infty, x, y \text { fixed }
$$

and their averages (3.5) are definitely zero. Thus the restriction imposed by (3.4) to the Kelvin wave profile can be written as

$$
\left\langle K_{w}^{(0)}(x-t)\right\rangle_{t}=\lim \frac{1}{T_{a v}} \int_{x-T_{a v}}^{x} K_{w}^{(0)}(z) d z=0, \quad \text { as } T_{a v} \rightarrow \infty .
$$

Applying (3.28) to (3.26) one obtains the simple formulae for the boundary value of the initial slow elevation

$\bar{h}_{0 I}^{(B)}=\int_{0}^{\infty}\left\langle u_{I}+h_{I}\right| e^{-y} d y$,

and the Kelvin wave profile:

$K_{w}^{(0)}(x)=\int_{0}^{\infty}\left[u_{I}+h_{I}-\left\langle u_{I}+h_{I}\right|\right] e^{-y} d y$

where the averaging

$\langle a(x)|=\lim \frac{1}{T_{a v}} \int_{x-T_{a v}}^{x} a(x) d x$, as $T_{a v} \rightarrow \infty$,

is introduced. Of course, if $a(x)$ is a smooth bounded function (as our initial fields are assumed to be) then the value $\langle a|$ does not depend on $x$ and depends only on the behaviour of $a(x)$ as $x \rightarrow-\infty$. 
Physically the condition (3.29) means that the fast Kelvin wave brings "information" from $x=-\infty$ to $x=+\infty$ propagating always in such a way that the boundary is to the right of the propagation direction. Correspondingly, the initial boundary condition for the slow component is determined only by the initial fields at $x=-\infty$.

Formula (3.30) gives us a simple way to calculate the lowest-order Kelvin wave profile for arbitrary initial conditions (2.2). In the case of periodic boundary conditions (2.9a) the condition (3.30) means that the periodic Kelvin wave profile should not contain a part independent of $x$ (purely zonal flow), i.e.

$$
K_{w}^{(0)}(x)=\int_{0}^{\infty}\left[u_{I}+h_{I}-u_{I 0}-h_{I 0}\right] e^{-y} d y
$$

and for $\bar{h}_{0 I}^{(B)}$ we have

$$
\bar{h}_{0 I}^{(B)}=\int_{0}^{\infty}\left(u_{I 0}+h_{I 0}\right) e^{-y} d y .
$$

In the case of an initial "zonal step" $(2.9 \mathrm{~b})$ the Kelvin wave profile and $\bar{h}_{0 I}^{(B)}$ are given by the formulae:

$$
\begin{aligned}
& K_{w}^{(0)}(x)=\int_{0}^{\infty}\left[u_{I}+h_{I}-u_{I}^{(-)}-h_{I}^{(-)}\right] e^{-y} d y \\
& \bar{h}_{0 I}^{(B)}=\int_{0}^{\infty}\left[u_{I}^{(-)}+h_{I}^{(-)}\right] e^{-y} d y
\end{aligned}
$$

Finally, in the localised case (2.9c) we have:

$$
\begin{aligned}
& K_{w}^{(0)}(x)=\int_{0}^{\infty}\left(u_{I}+h_{I}\right) e^{-y} d y, \\
& \bar{h}_{0 I}^{(B)}=0 .
\end{aligned}
$$

The Kelvin wave profiles for each of the above cases are schematically shown in Fig. 2.

Note that in the case of geostrophically balanced initial conditions when $h_{I}=\bar{h}_{0 I}=0$ and $u_{I}=-\partial h_{I} / \partial y$ the Kelvin wave disappears as follows from (3.26). In the absence of nonlinearity the initial Kelvin wave profile (3.30) propagates steadily; the nonlinearity forces the profile to change slowly in time (see below Section 5).

Knowing the boundary condition (3.29) (or (3.32b), (3.33b), (3.34b)) one can find $\bar{h}_{0 /}$ using the equation

$$
\nabla^{2} \bar{h}_{0 I}-\bar{h}_{0 I}=\Pi_{I}=\frac{\partial v_{I}}{\partial x}-\frac{\partial u_{I}}{\partial y}-h_{I}
$$


which readily follows from the initial conditions (3.1e) and equations (3.3), (3.6c), (3.7c). Obviously, the solution $\bar{h}_{0 I}, \tilde{u}_{0}, \tilde{v}_{0}, \tilde{h}_{0}$ (without the slow time dependence) describes the linear adjustment of an arbitrary initial field (2.2) on the half-plane.

Thus, in the lowest order we get a fast-slow motion splitting defined in a unique way for arbitrary initial conditions. Note that the procedure imposes no limitations on the relative initial values of fast and slow components. The fast part of the motion is completely resolved while the slow one remains undetermined. The slow evolution is determined from the condition of the absence of secular growth of the first-order solution. 


\section{Dynamics of the lowest-order slow motion}

\section{Problem for the lowest-order slow motion}

To describe the time development of the lowest-order slow component and slow evolution of the fast one we consider the first-order solution. Substitution of $(2.10)$ into $(2.1 \mathrm{a}, \mathrm{b}),(2.2),(2.3)$, (2.5) gives

$$
\begin{aligned}
& \frac{\partial u_{1}}{\partial t}-v_{1}=-\frac{\partial h_{1}}{\partial x}+R_{u}^{(1)}, \quad \frac{\partial v_{1}}{\partial t}+u_{1}=-\frac{\partial h_{1}}{\partial y}+R_{v}^{(1)}, \quad \frac{\partial\left(\varsigma_{1}-h_{1}\right)}{\partial t}=R_{\varsigma}^{(1)}, \\
& R_{u}^{(1)}=-\left(\frac{\partial u_{0}}{\partial T_{1}}+u_{0} \frac{\partial u_{0}}{\partial x}+v_{0} \frac{\partial u_{0}}{\partial y}\right), \quad R_{v}^{(1)}=-\left(\frac{\partial v_{0}}{\partial T_{1}}+u_{0} \frac{\partial v_{0}}{\partial x}+v_{0} \frac{\partial v_{0}}{\partial y}\right), \\
& R_{\varsigma}^{(1)}=-\frac{\partial\left(\varsigma_{0}-h_{0}\right)}{\partial T_{1}}-\frac{\partial\left[u_{0}\left(\varsigma_{0}-h_{0}\right)\right]}{\partial x}-\frac{\partial\left[v_{0}\left(\varsigma_{0}-h_{0}\right)\right]}{\partial y}, \\
& \left.v_{1}\right|_{y=0}=0, \quad\left(u_{1}, v_{1}, h_{1}\right)_{t=0}=0 .
\end{aligned}
$$

Analysis of system (4.1) is carried out along the same lines as in the lowest-order case. We represent the first-order field as a sum of fast and slow components,

$\left(u_{1}, v_{1}, h_{1}\right)=\left(\widetilde{u}_{1}, \widetilde{v}_{1}, \widetilde{h}_{1}\right)\left(x, y, t, T_{1}, \ldots\right)+\left(u_{1}, \nabla_{1}, h_{1}\right)\left(x, y, T_{1}, \ldots\right)$,

the fast component having zero average (3.5), and apply (3.5) to equations (4.1).

The averaging of the first-order vorticity equation (4.1c) gives the QG potential vorticity equation

$$
\frac{\partial\left(\nabla^{2} \bar{h}_{0}-\bar{h}_{0}\right)}{\partial T_{1}}+J\left(\bar{h}_{0}, \nabla^{2} \bar{h}_{0}\right)=0
$$

describing evolution of the slow motion. The first-order slow equations are written in the form

$$
\begin{aligned}
& \bar{v}_{1}=\frac{\partial \bar{h}_{1}}{\partial x}-\bar{R}_{u}^{(1)}, \quad \bar{u}_{1}=-\frac{\partial \bar{h}_{1}}{\partial y}+\bar{R}_{v}^{(1)}, \quad \bar{\varsigma}_{1}-\bar{h}_{1}=\Pi_{1}\left(x, y, T_{1}, \ldots\right), \\
& \left.\bar{v}_{1}\right|_{y=0}=0 ; \\
& \bar{R}_{u}^{(1)}=-\left(\frac{\partial \bar{u}_{0}}{\partial T_{1}}+\bar{u}_{0} \frac{\partial \bar{u}_{0}}{\partial x}+\bar{v}_{0} \frac{\partial \bar{u}_{0}}{\partial y}\right), \quad \bar{R}_{v}^{(1)}=-\left(\frac{\partial \bar{v}_{0}}{\partial T_{1}}+\bar{u}_{0} \frac{\partial \bar{v}_{0}}{\partial x}+\bar{v}_{0} \frac{\partial \bar{v}_{0}}{\partial y}\right) .
\end{aligned}
$$

Here $\Pi_{1}$ is some function of the slow variables which is determined at the next order. Note that the averages of the nonlinear terms containing the lowest-order fast fields $\tilde{u}_{0}, \tilde{v}_{0}$ are zero, which readily follows from the large-time decay of the lowest-order fast fields (see (3.27)) and the property (3.28) of Kelvin wave. 
The initial field $\bar{h}_{0 I}$ is determined by equations (3.35), (3.29), and the boundary value of $\bar{h}_{0}$ depends only on the slow time by virtue of $(3.6 \mathrm{a}, \mathrm{d})$,

$\left.\bar{h}_{0}\right|_{y=0}=\bar{h}_{0 B}\left(T_{1}, \ldots\right)$.

To find the function $\bar{h}_{0 B}\left(T_{1}, \ldots\right)$ in $(4.5)$ we use the boundary condition for the first-order slow variable $\bar{h}_{1}$ that readily follows from equations $(4.4 \mathrm{a}),(4.4 \mathrm{~d})$, and $(4.4 \mathrm{e})$ :

$\left.\frac{\partial \bar{h}_{1}}{\partial x}\right|_{y=0}=-\left(\frac{\partial \bar{u}_{0}}{\partial T_{1}}+\bar{u}_{0} \frac{\partial \bar{u}_{0}}{\partial x}\right)_{y=0}$.

The function $\bar{h}$ should be bounded as $x \rightarrow \pm \infty$, therefore the condition (4.6) imposes the following additional restriction to the lowest-order fields:

$\left\langle\left.\frac{\partial \bar{u}_{0}}{\partial T_{1}}\right|_{y=0}\right\rangle_{x}=-\left\langle\left.\frac{\partial^{2} \bar{h}_{0}}{\partial y \partial T_{1}}\right|_{y=0}\right\rangle_{x}=0$,

where the averaging \langle\rangle$_{x}$ is determined as

$\langle a\rangle_{x}=\lim \frac{1}{2 L} \int_{-L}^{L} a d x, \quad L \rightarrow \infty$.

The condition (4.7) of constant mean circulation along the boundary is well-known (see e.g. Kamenkovich \& Reznik 1978, Pedlosky 1987) but previously it was obtained for geostrophically balanced initial conditions (2.2), i.e. no fast component was present in the system.

\section{Solvability of the problem for $\bar{h}_{0}$}

The problem (4.3), (4.5), and (4.7) together with initial field $\bar{h}_{\mathrm{O} /}$ known from (3.29), (3.35) is complete and allows us to determine the lowest-order slow geostrophic component of the motion. To demonstrate the solvability of this problem we rewrite (4.3) in the form

$\nabla^{2} D-D=R_{D}, \quad D=\frac{\partial \bar{h}_{0}}{\partial T_{1}}, \quad R_{D}=-J\left(\bar{h}_{0}, \nabla^{2} \bar{h}_{0}\right)$,

and assume $\bar{h}_{0}$ to be known at some moment $T_{1}=T_{10}$. We now represent $D$ as a sum

$D=D_{0}+D_{1}$

where $D_{0}, D_{1}$ satisfy the equations:

$\nabla^{2} D_{0}-D_{0}=R_{D},\left.\quad D_{0}\right|_{y=0}=0 ;$

$\nabla^{2} D_{1}-D_{1}=0,\left.\quad D_{1}\right|_{\mathrm{y}=0}=\frac{\partial \bar{h}_{0 B}}{\partial T_{1}}$. 
Here $R_{D}$ and, therefore, $D_{0}$ are known by the above assumption, and so $\frac{\partial \bar{h}_{0 B}}{\partial T_{1}}$ can be found. Since $\frac{\partial \bar{h}_{0 B}}{\partial T_{1}}$ does not depend on $x$ we have

$D_{1}=\frac{\partial \bar{h}_{0 B}}{\partial T_{1}} e^{-y}$

and substitution of (4.10) into the condition (4.7) gives

$$
\frac{\partial \bar{h}_{0 B}}{\partial T_{1}}=\left\langle\left.\frac{\partial D_{0}}{\partial y}\right|_{y=0}\right\rangle_{x}
$$

whence

$$
\frac{\partial \bar{h}_{0}}{\partial T_{1}}=D_{0}+e^{-y}\left\langle\left.\frac{\partial D_{0}}{\partial y}\right|_{y=0}\right\rangle_{x}
$$

Given $\frac{\partial \bar{h}_{0}}{\partial T_{1}}$ one can calculate the field $\bar{h}_{0}$ at $T_{1}=T_{10}+d T_{1}$, and so on.

For the zonal "step" case (2.9b) the boundary condition (4.5) (i.e. the function $\bar{h}_{O B}$ ) does not depend on time and the slow motion does not change as $x \rightarrow \mp \infty$ being approximately zonal there. To show this, we note that if at some moment $T_{1}=T_{10}$

$\bar{h}_{0} \rightarrow \bar{h}_{0}^{ \pm}(y), \quad x \rightarrow \pm \infty$

then the derivative $\frac{\partial \bar{h}_{0}}{\partial x}$ and Jacobian $R_{D}$ are localised in $x$. In this case the solution $D_{0}$ to the problem (4.11) is also localised in $x$ i.e. $\left\langle\left.\frac{\partial D_{0}}{\partial y}\right|_{y=0}\right\rangle_{x}=0$. It means that $\frac{\partial \bar{h}_{0 B}}{\partial T_{1}}=0$ (see (4.14)) and the derivative $\frac{\partial \bar{h}_{0}}{\partial T_{1}}=D_{0}$ is also localised in $x$ at the moment $T_{1}=T_{10}$. This means that $\bar{h}_{0}$ does not change as $x \rightarrow \mp \infty$ and the property (4.16) remains valid at the moment $T_{1}=T_{10}+d T_{1}$ and so on. By virtue of (2.9b) we have for the initial vorticity $\Pi_{I}$ (right-hand side of equation (3.35)):

$\Pi_{I} \rightarrow-\frac{\partial u_{I}^{( \pm)}(y)}{\partial y}-h_{I}^{( \pm)}(y)$, as $x \rightarrow \pm \infty$,

therefore the initial field $\bar{h}_{0 I}$ also obeys (4.16). Thus for the zonal step initial condition the slow component remains unchanged at $x=\mp \infty$, and is described by the QG equation (4.3) with the timeindependent boundary condition

$\bar{h}_{0 B}=\int_{0}^{\infty}\left[u_{I}^{(-)}+h_{I}^{(-)}\right] e^{-y} d y=$ constant.

This consideration remains valid for the localized initial conditions (2.9c) which are special, but a practically important case of the "step" initial conditions. Evolution of the localized initial field $\bar{h}_{0 /}$ is governed by (4.3) under the condition 
$\bar{h}_{0 B}=0$

and $\bar{h}_{0}$ remains localised for all time. It means that that the right-hand side of (4.6) is also localised and the first-order correction $\bar{h}_{1}$ is always bounded. Correspondingly, the condition (4.7) for the localised case is superfluous since it is satisfied identically, and imposes no additional restrictions to the motion.

\section{Mass, energy, and enstrophy conservation}

Mass and energy conservation for the periodic and "step" cases are obtained in the usual way, and have the forms

$$
\frac{\partial}{\partial T_{1}} \int_{0}^{\infty}\left\langle\overline{h_{0}}\right\rangle_{x} d y=-\left\langle\left.\frac{\partial^{2} \bar{h}_{0}}{\partial y \partial T_{1}}\right|_{y=0}\right\rangle_{x}=0
$$

and

$$
\frac{\partial}{\partial T_{1}} \int_{0}^{\infty}\left\langle\frac{1}{2}\left[\left(\nabla \bar{h}_{0}\right)^{2}+\bar{h}_{0}^{2}\right]\right\rangle_{x} d y=-\bar{h}_{0 B}\left\langle\left.\frac{\partial^{2} \bar{h}_{0}}{\partial y \partial T_{1}}\right|_{y=0}\right\rangle_{x}=0,
$$

respectively. Thus the condition (4.7) provides the energy and mass conservation for the lowestorder slow motion. Multiplying (4.3) by $\nabla^{2} \bar{h}_{0}$, averaging in $x$ and integrating in $y$ from 0 to $\infty$ we obtain the enstrophy conservation law

$\frac{\partial}{\partial T_{1}}\left\{\int_{0}^{\infty}\left\langle\frac{1}{2}\left[\left(\nabla \bar{h}_{0}\right)^{2}+\left(\nabla^{2} \bar{h}_{0}\right)^{2}\right]\right\rangle_{x} d y+\Gamma \bar{h}_{0 B}\right\}=0$,

where $\Gamma=\left\langle\left.\frac{\partial \bar{h}_{0}}{\partial y}\right|_{y=0}\right\rangle_{x}=$ constant by virtue of (4.7). Note that the law (4.20c) includes the extra boundary term $\Gamma \bar{h}_{0 B}$ absent in an unbounded fluid.

For the localised case, the energy and enstrophy conservation laws have a standard form, and the mass conservation is written as

$$
\frac{\partial}{\partial T_{1}} \int_{-\infty}^{\infty} d x \int_{0}^{\infty} \overline{h_{0}} d y=-\left.\int \frac{\partial^{2} \bar{h}_{0}}{\partial y \partial T_{1}}\right|_{y=0} d x
$$

It readily follows from (4.21) that the mass is conserved only under the condition of zero alongboundary circulation,

$$
\left.\int_{-\infty}^{\infty} \frac{\partial^{2} \bar{h}_{0}}{\partial y \partial T_{1}}\right|_{y=0} d x=0
$$


Note that the equation (4.22) is also a condition for locality of the first-order slow correction $\bar{h}_{1}$, as is readily seen from (4.6). But the problem (4.3), (4.19) is well-posed and the condition (4.22) turns out to be superfluous, i.e. it can contradict (4.19). To demonstrate this we use the formula

$$
\left.\int_{-\infty}^{\infty} \frac{\partial^{2} \bar{h}_{0}}{\partial y \partial T_{1}}\right|_{y=0} d x=\int_{-\infty}^{\infty} d x \int_{0}^{\infty} \frac{\partial \bar{h}_{0}}{\partial x} \frac{\partial \bar{h}_{0}}{\partial y} e^{-y} d y
$$

which follows from (4.3) and (4.19). To derive (4.23) we integrate (4.9) over all $x$, and find the function $H=\int_{-\infty}^{\infty} \frac{\partial \overline{h_{0}}}{\partial T_{1}} d x$, from the resulting equation, with the boundary condition $\left.H\right|_{y=0}=0$.

Generally, the integral on the right hand side of (4.23) does not vanish for an arbitrary localised $\bar{h}_{0}$ satisfying (4.19). It means that the condition (4.22) can be violated, at least at the initial moment $T_{1}=0$. Therefore equations (4.3), (4.19), generally, do not provide validity of (4.22), which means (somewhat unexpectedly) that in the case of localised initial conditions (2.9c): 1) the mass of the slow localised motion on a bounded half-plane may not be conserved; 2) the first-order slow correction field may not be localised.

\section{First-order solution}

\section{Problem for the fast first-order component}

For the fast first-order components we have (cf. RZB):

$$
\begin{aligned}
& \frac{\partial \tilde{u}_{1}}{\partial t}-\tilde{v}_{1}=-\frac{\partial \tilde{h}_{1}}{\partial x}+\tilde{R}_{u}^{(1)}, \quad \frac{\partial \tilde{v}_{1}}{\partial t}+\tilde{u}_{1}=-\frac{\partial \tilde{h}_{1}}{\partial y}+\tilde{R}_{v}^{(1)}, \quad \tilde{\zeta}_{1}-\tilde{h}_{1}=\tilde{R}_{\varsigma}^{(1)}, \\
& \left.\tilde{v}_{1}\right|_{y=0}=0 .
\end{aligned}
$$

Here

$$
\begin{aligned}
& \tilde{R}_{u}^{(1)}=-\left(\frac{\partial \tilde{u}_{0}}{\partial T_{1}}+\tilde{u}_{0} \frac{\partial \tilde{u}_{0}}{\partial x}+\tilde{v}_{0} \frac{\partial \tilde{u}_{0}}{\partial y}+\bar{u}_{0} \frac{\partial \tilde{u}_{0}}{\partial x}+\bar{v}_{0} \frac{\partial \tilde{u}_{0}}{\partial y}+\tilde{u}_{0} \frac{\partial \bar{u}_{0}}{\partial x}+\tilde{v}_{0} \frac{\partial \bar{u}_{0}}{\partial y}\right) \\
& \tilde{R}_{v}^{(1)}=-\left(\frac{\partial \tilde{v}_{0}}{\partial T_{1}}+\tilde{u}_{0} \frac{\partial \tilde{v}_{0}}{\partial x}+\tilde{v}_{0} \frac{\partial \tilde{v}_{0}}{\partial y}+\bar{u}_{0} \frac{\partial \tilde{v}_{0}}{\partial x}+\bar{v}_{0} \frac{\partial \tilde{v}_{0}}{\partial y}+\tilde{u}_{0} \frac{\partial \bar{v}_{0}}{\partial x}+\tilde{v}_{0} \frac{\partial \bar{v}_{0}}{\partial y}\right) \\
& \tilde{R}_{\varsigma}^{(1)}=-\frac{\partial\left[\tilde{U}_{0}\left(\varsigma_{0}-h_{0}\right)\right]}{\partial x}-\frac{\partial\left[\tilde{V}_{0}\left(\varsigma_{0}-h_{0}\right)\right]}{\partial y}, \\
& \tilde{U}_{0}=\tilde{U}_{0 i}-\left\langle\tilde{U}_{0 i}\right\rangle_{t}, \quad \tilde{V}_{0}=\tilde{V}_{0 i}-\left\langle\tilde{V}_{0 i}\right\rangle_{t}, \quad\left(\tilde{U}_{0 i}, \tilde{V}_{0 i}\right)=\int_{0}^{t}\left(\tilde{u}_{0}, \tilde{v}_{0}\right) d t .
\end{aligned}
$$


To derive equation (5.1c) we integrate (4.1c) over $t$ from 0 to $t$ taking into account (4.1h) and, using the averaging procedure (3.5), split the resulting equation into the slow and fast parts. The function $\tilde{V}_{0}$ is determined from the system

$L_{w} \tilde{V}_{0}=0,\left.\quad \tilde{V}_{0}\right|_{y=0}=0,\left.\quad \tilde{V}_{0}\right|_{t=0}=-\left\langle\tilde{V}_{0 i}\right\rangle_{t},\left.\quad \frac{\partial \tilde{V}_{0}}{\partial t}\right|_{t=0}=F(x, y)$,

where, in turn, $\left\langle\tilde{V}_{0 i}\right\rangle_{t}$ obeys the equations

$$
\nabla^{2}\left\langle\tilde{V}_{0 i}\right\rangle_{t}-\left\langle\tilde{V}_{0 i}\right\rangle_{t}=-G(x, y),\left.\left\langle\tilde{V}_{0 i}\right\rangle_{t}\right|_{y=0}=0
$$

Given $\tilde{V}_{0}$ the function $\tilde{U}_{0}$ is determined by the formulae

$\tilde{U}_{0}=\tilde{U}_{01}+\tilde{U}_{0 k}, \quad \frac{\partial U_{0 k}}{\partial t}=K_{w}^{(0)}(x-t) e^{-y}$,

where

$\tilde{U}_{01}=-\frac{s^{+}}{2}-\frac{1}{2} \frac{\partial}{\partial x}\left(e^{y} \int_{y}^{\infty} \tilde{V}_{0} e^{-y} d y-e^{-y} \int_{-\infty}^{y} \tilde{V}_{0} e^{y} d y\right)$.

The function $\tilde{U}_{0 k}$ is given by the equation

$\tilde{U}_{0 k}=-e^{-y} \int_{-\infty}^{x-t} K_{w}^{(0)}\left(z, T_{1}, \ldots\right) d z$

in the "step" and localised cases, and

$\tilde{U}_{0 k}=i e^{-y} \sum_{m} \frac{k_{m}\left(T_{1}, \ldots\right)}{m} e^{i m(x-t)}, k_{0}=0$,

in the periodic case; here $k_{m}$ are the coefficient in the Fourier-series expansion in $x$ of the Kelvin wave $K_{w}^{(0)}\left(x-t, T_{1}, \ldots\right) e^{-y}$.

Initial conditions for the fast and slow first-order zonal velocities

Like the lowest-order approximation (Section 3) the analysis starts with calculating the initial slow first-order meridional velocity $\bar{v}_{1 I}$. At first, we derive from $(4.4 \mathrm{a}, \mathrm{b}, \mathrm{c})$ the equation for $\frac{\partial \bar{h}_{1 I}}{\partial x}$ analogous to (3.8),

$\nabla^{2} \frac{\partial \bar{h}_{1 I}}{\partial x}-\frac{\partial \bar{h}_{1 I}}{\partial x}=\frac{\partial}{\partial x}\left[\Pi_{1 I}+\operatorname{div} \overline{\mathbf{R}}_{\mathbf{v} I}^{(1)}\right]$

where 
$\overline{\mathbf{R}}_{\mathbf{v}}^{(1)}=\left(\bar{R}_{u}^{(1)}, \bar{R}_{v}^{(1)}\right), \quad \operatorname{div} \overline{\mathbf{R}}_{\mathbf{v}}^{(1)}=2\left[\frac{\partial^{2} \bar{h}_{0}}{\partial x^{2}} \frac{\partial^{2} \bar{h}_{0}}{\partial y^{2}}-\left(\frac{\partial^{2} \bar{h}_{0}}{\partial x \partial y}\right)^{2}\right]$

The boundary condition for (5.5a) follows from (4.6):

$\left.\frac{\partial \bar{h}_{1 I}}{\partial x}\right|_{y=0}=-\left(\left.\frac{\partial \bar{u}_{0}}{\partial T_{1}}\right|_{t=0}+\bar{u}_{0 I} \frac{\partial \bar{u}_{0 I}}{\partial x}\right)_{y=0}$

To make the problem (5.5a), (5.6) complete one should find $\Pi_{1 / l}$ and $\left.\frac{\partial \bar{u}_{0}}{\partial T_{1}}\right|_{t=0}$. By virtue of (4.4c), (5.1c) $\varsigma_{1}-h_{1}=\Pi_{1}+\tilde{R}_{\varsigma}^{(1)}$, and, therefore, taking into account initial conditions (4.1h), we have:

$$
\Pi_{1 I}=-\tilde{R}_{\zeta I}^{(1)}=\frac{\partial\left(\tilde{U}_{0 I} \Pi_{I}\right)}{\partial x}+\frac{\partial\left(\tilde{V}_{0 I} \Pi_{I}\right)}{\partial y}
$$

where $\tilde{U}_{0 I}, \tilde{V}_{0 I}$ can be found using (5.2) to (5.4). The derivative $\left.\frac{\partial \bar{u}_{0}}{\partial T_{1}}\right|_{t=0}$ is obtained from (4.15), $\left.\frac{\partial \bar{u}_{0}}{\partial T_{1}}\right|_{t=0}=-\left.\frac{\partial^{2} \bar{h}_{0}}{\partial y \partial T_{1}}\right|_{t=0}=-\frac{\partial D_{0 I}}{\partial y}+e^{-y}\left\langle\left.\frac{\partial D_{0 I}}{\partial y}\right|_{y=0}\right\rangle_{x}$

Thus the problem for $\frac{\partial \bar{h}_{1 I}}{\partial x}$ is well-defined and given $\frac{\partial \bar{h}_{1 I}}{\partial x}$ the initial field $\bar{v}_{1 I}$ can be determined from $(4.4 \mathrm{a}, \mathrm{e})$ :

$$
\bar{v}_{1 I}=\frac{\partial \bar{h}_{1 I}}{\partial x}-\bar{R}_{u I}^{(1)} .
$$

The function $\bar{v}_{1 I}$ is periodic for the periodic case (2.9a), and localised for the cases $(2.9 \mathrm{~b}, \mathrm{c})$. Given $\bar{v}_{1 I}$ one can determine one initial condition $\tilde{v}_{1 I}$ from $(4.1 \mathrm{~h})$,

$$
\tilde{v}_{1 I}=-\bar{v}_{1 I} \text {. }
$$

Another initial condition for $\widetilde{v}_{1}$ follows from $(4.1 \mathrm{~b}, \mathrm{~h})$ :

$$
\left.\frac{\partial \tilde{v}_{1}}{\partial t}\right|_{t=0}=R_{v I}^{(1)}=-\left(\left.\frac{\partial v_{0}}{\partial T_{1}}\right|_{t=0}+u_{I} \frac{\partial v_{I}}{\partial x}+v_{I} \frac{\partial v_{I}}{\partial y}\right) .
$$

As we will see below, $\tilde{v}_{0}$ does not depend on the slow time $T_{1}$ therefore from (4.15):

$$
\left.\frac{\partial v_{0}}{\partial T_{1}}\right|_{t=0}=\left.\frac{\partial^{2} \bar{h}_{0}}{\partial x \partial T_{1}}\right|_{t=0}=\frac{\partial D_{0 I}}{\partial x} .
$$

Analysis of resonances in the equation for $\tilde{v}_{1}$

The equation for $\tilde{v}_{1}$ follows from $(5.1 \mathrm{a}, \mathrm{b}, \mathrm{c})$ (see RZB for more details):

$-\frac{\partial^{2} \tilde{v}_{1}}{\partial t^{2}}+\nabla^{2} \tilde{v}_{1}-\tilde{v}_{1}=\tilde{F}_{v}^{(1)}$ 


$$
\tilde{F}_{v}^{(1)}=-\left(\frac{\partial^{2} \tilde{R}_{u}^{(1)}}{\partial y^{2}}-\tilde{R}_{u}^{(1)}+\frac{\partial \tilde{R}_{v}^{(1)}}{\partial t}-\frac{\partial^{2} \tilde{R}_{v}^{(1)}}{\partial x \partial y}+\frac{\partial^{2} \tilde{R}_{\varsigma}^{(1)}}{\partial t \partial y}-\frac{\partial \tilde{R}_{\varsigma}^{(1)}}{\partial x}\right)
$$

This equation together with the initial conditions (5.10), (5.11) and the boundary condition (5.1d) determine the field $\widetilde{v}_{1}$.

Of course, the first question is if the right-hand side part of (5.13a) contains secular terms causing either a rapidly growing response, or a response with a non-zero average (3.5) (in this case our first-order splitting (4.2) fails). To examine this issue the source term $\tilde{F}_{v}^{(1)}$ is represented as a sum (using (3.15b), cf. RZB)

$$
\tilde{F}_{v}^{(1)}=2 \frac{\partial^{2} \tilde{v}_{0}}{\partial T_{1} \partial t}+\Phi_{0}^{(s)} \Phi_{0}^{(i g)}+\Phi_{1}^{(i g)} \Phi_{2}^{(i g)}+\Phi_{0}^{(k)} \Phi_{3}^{(i g)}+\Phi_{1}^{(k)} \Phi_{1}^{(s)}+\Phi_{2}^{(k)} \Phi_{3}^{(k)}
$$

where the superscripts " $s$ ", "ig", and " $k$ " denote the slow component, the component consisting of the IG waves, and Kelvin wave component, respectively. Each of the functions $\Phi_{m}^{(i g)}, \Phi_{m}^{(k)}, m=0,1,2,3$ is a solution to the homogeneous wave equation (3.11); the slow functions $\Phi_{n}^{(s)}, n=0,1$ do not depend on the fast time $t$. The functions $\Phi_{n}^{(s)}, \Phi_{m}^{(i g)}, \Phi_{m}^{(k)}$ are localised in the $y$ direction, and either periodic or bounded or localised in $x$-direction depending on the initial conditions (2.9). Analysis of all possible interactions is rather tedious; some estimates for the localised case are given in Appendix B. Here only the results will be reported.

Triad interactions between IG waves are prohibited, and, therefore the response $\tilde{v}_{w w}$ generated by the term $\Phi_{1}^{(i g)} \Phi_{2}^{(i g)}$ decays rapidly, $\left.\tilde{v}_{w w}\right|_{t=\infty}=O\left(t^{-3}\right)$ at a fixed point $x, y$. The interactions between Kelvin waves are contained in the first two terms in the brackets in the right hand side of (5.13b) and the corresponding source term $\Phi_{2}^{(k)} \Phi_{3}^{(k)}$ has the form

$$
\Phi_{2}^{(k)} \Phi_{3}^{(k)}=3 K_{w}^{(0)}\left(x-t, T_{1}, \ldots\right) \frac{\partial K_{w}^{(0)}}{\partial x}\left(x-t, T_{1}, \ldots\right) e^{-2 y} .
$$

The corresponding response

$$
\tilde{v}_{k k}=K_{w}^{(0)}\left(x-t, T_{1}, \ldots\right) \frac{\partial K_{w}^{(0)}}{\partial x}\left(x-t, T_{1}, \ldots\right) e^{-2 y}
$$

does not decay in time but it is fast since $\left\langle\tilde{v}_{k k}\right\rangle_{t}=0$.

and makes no contribution to the slow component.

The responses $\tilde{v}_{s w}$ and $\tilde{v}_{k w}$ to the interactions between the slow component and IG waves (term $\Phi_{0}^{(s)} \Phi_{0}^{(i g)}$ ), and between Kelvin and IG waves (term $\Phi_{0}^{(k)} \Phi_{3}^{(i g)}$ ), respectively, decay in time but the decay rate depends on the initial conditions. For the periodic initial conditions both $\tilde{v}_{s w}$ and $\tilde{v}_{k w}$ 
are $O\left(t^{-1 / 2}\right), t \rightarrow \infty$, while for "step" and localised cases $\tilde{v}_{s w}=O\left(t^{-1}\right), \tilde{v}_{k w}=O\left(t^{-3 / 2}\right)$. Finally, the response $\tilde{v}_{s k}$ to the interaction between Kelvin waves and the slow motion (term $\Phi_{1}^{(k)} \Phi_{1}^{(s)}$ ) does not decay for the periodic case, but $\left\langle\tilde{v}_{s k}\right\rangle_{t}=0$, and $\tilde{v}_{s k}=O\left(t^{-1}\right)$ for the "step" and localised cases. We note that although the Fourier representations of the non-linear terms $\Phi_{0}^{(s)} \Phi_{0}^{(i g)}, \Phi_{0}^{(k)} \Phi_{3}^{(i g)}$, and $\Phi_{1}^{(k)} \Phi_{1}^{(s)}$ contain resonant harmonics their measure is zero in the continuous spectrum and these harmonics give no dangerous contributions (cf. RZB).

The general conclusion of this analysis is that the response to nonlinear interactions in (5.14) does not grow in time, and it is fast in the sense that it has a zero time-average (3.5). We emphasise that taking into account the boundary and initial conditions (5.1d), (5.10), (5.11) does not change this conclusion.

The lack of the resonant non-linear terms in the right hand side of (5.13a) means that $\tilde{v}_{0}$, and, therefore, the function $\tilde{v}_{00}$ in (3.14) do not depend on the slow time $T_{1}$ since in the opposite case the first term in the right hand side of (5.14) gives a secular growth. Obviously, the functions $\tilde{u}_{01}, \tilde{h}_{01}$ in (3.16) also do not depend on $T_{1}$, since they linearly depend on $\tilde{v}_{0}$ (see Sec. 3). At the same time, as we will see below, the lowest-order Kelvin wave in (3.16) does depend on slow times to prevent secular growth of the first-order Kelvin wave.

\section{The fields $\tilde{u}_{1}, \tilde{h}_{1}$, and the first-order Kelvin wave}

The fields $\tilde{u}_{1}, \tilde{h}_{1}$ are found analogously to $\tilde{u}_{0}, \tilde{h}_{0}$ in Sec. 3 . The equations analogous to (3.15) simply follow from $(5.1 \mathrm{a}, \mathrm{b}, \mathrm{c})$,

$$
\begin{aligned}
& \frac{\partial^{2} \tilde{h}_{1}}{\partial y^{2}}-\tilde{h}_{1}=\tilde{F}_{h}^{(1)}=-\left(\frac{\partial \tilde{v}_{1}}{\partial x}+\frac{\partial^{2} \tilde{v}_{1}}{\partial t \partial y}\right)+\tilde{R}_{\varsigma}^{(1)}+\frac{\partial \tilde{R}_{v}^{(1)}}{\partial y}, \\
& \frac{\partial^{2} \tilde{u}_{1}}{\partial y^{2}}-\tilde{u}_{1}=\tilde{F}_{u}^{(1)}=\frac{\partial \tilde{v}_{1}}{\partial t}+\frac{\partial^{2} \tilde{v}_{1}}{\partial x \partial y}-\tilde{R}_{v}^{(1)}-\frac{\partial \tilde{R}_{\varsigma}^{(1)}}{\partial y},
\end{aligned}
$$

Solutions to $(5.18 \mathrm{a}, \mathrm{b})$ are conveniently written in the form

$$
\tilde{u}_{1}=\tilde{u}_{11}+C_{u}^{(1)}\left(x, t, T_{1}, \ldots\right) e^{-y}, \quad \tilde{h}_{1}=\tilde{h}_{11}(x, y, t)+C_{h}^{(1)}\left(x, t, T_{1}, \ldots\right) e^{-y} .
$$

Here $C_{u}^{(1)}$ and $C_{h}^{(1)}$ are some arbitrary functions which have to be determined and

$$
\tilde{u}_{11}=-\frac{1}{2}\left(e^{y} \int_{y}^{\infty} \tilde{F}_{u}^{(1)} e^{-y} d y+e^{-y} \int_{0}^{y} \tilde{F}_{u}^{(1)} e^{y} d y\right),
$$


$\tilde{h}_{11}=-\frac{1}{2}\left(e^{y} \int_{y}^{\infty} \tilde{F}_{h}^{(1)} e^{-y} d y+e^{-y} \int_{0}^{y} \tilde{F}_{h}^{(1)} e^{y} d y\right)$.

To determine $C_{u}^{(1)}$ and $C_{h}^{(1)}$ we write (5.1a,b,c) at $y=0$ (cf. derivation of (3.22)):

$\frac{\partial \tilde{u}_{1}}{\partial t}+\frac{\partial \tilde{h}_{1}}{\partial x}=\left.\tilde{R}_{u}^{(1)}\right|_{y=0}, \quad \tilde{u}_{1}=-\frac{\partial \tilde{h}_{1}}{\partial y}, \quad \tilde{h}_{1}=-\frac{\partial \tilde{u}_{1}}{\partial y}-\left.\tilde{R}_{\varsigma}^{(1)}\right|_{y=0}, \quad y=0$.

By virtue of (5.19) we have

$$
\begin{aligned}
& \left(\tilde{u}_{1}, \tilde{h}_{1}\right)_{y=0}=-\frac{1}{2} \int_{0}^{\infty}\left(\tilde{F}_{u}^{(1)}, \tilde{F}_{h}^{(1)}\right) e^{-y} d y+\left(C_{u}^{(1)}, C_{h}^{(1)}\right), \\
& \left(\frac{\partial \tilde{u}_{1}}{\partial y}, \frac{\partial \tilde{h}}{\partial y}\right)_{y=0}=-\frac{1}{2} \int_{0}^{\infty}\left(\tilde{F}_{u}^{(1)}, \tilde{F}_{h}^{(1)}\right) e^{-y} d y-\left(C_{u}^{(1)}, C_{h}^{(1)}\right) .
\end{aligned}
$$

Substitution of (5.22) into $(5.21 \mathrm{~b}, \mathrm{c})$ gives

$$
\begin{aligned}
& C_{u}^{(1)}-C_{h}^{(1)}=\frac{1}{2} \int_{0}^{\infty}\left(\tilde{F}_{u}^{(1)}+\tilde{F}_{h}^{(1)}\right) e^{-y} d y, \\
& C_{u}^{(1)}-C_{h}^{(1)}=-\frac{1}{2} \int_{0}^{\infty}\left(\tilde{F}_{u}^{(1)}+\tilde{F}_{h}^{(1)}\right) e^{-y} d y+\left.\tilde{R}_{\varsigma}^{(1)}\right|_{y=0} .
\end{aligned}
$$

The equations (5.23) are compatible since

$\int_{0}^{\infty}\left(\tilde{F}_{u}^{(1)}+\tilde{F}_{h}^{(1)}\right) e^{-y} d y=\left.\tilde{R}_{\varsigma}^{(1)}\right|_{y=0}$.

The validity of (5.24) can be shown using the identity

$$
\tilde{F}_{u}^{(1)}+\tilde{F}_{h}^{(1)}=\frac{\partial F}{\partial y}-F, \quad F=\frac{\partial \tilde{v}_{1}}{\partial x}-\frac{\partial \tilde{v}_{1}}{\partial t}+\tilde{R}_{v}^{(1)}-\tilde{R}_{\varsigma}^{(1)} .
$$

Thus we have

$$
C_{u}^{(1)}=C_{h}^{(1)}+\left.\frac{1}{2} \tilde{R}_{\varsigma}^{(1)}\right|_{y=0} .
$$

Substituting (5.22a), (5.26) into (5.21a) we obtain the equation for $C_{h}^{(1)}$ :

$$
\frac{\partial C_{h}^{(1)}}{\partial t}+\frac{\partial C_{h}^{(1)}}{\partial x}=\frac{1}{2} \int_{0}^{\infty}\left(\frac{\partial \tilde{F}_{u}^{(1)}}{\partial t}+\frac{\partial \tilde{F}_{h}^{(1)}}{\partial x}\right) e^{-y} d y+\left.\tilde{R}_{u}^{(1)}\right|_{y=0}-\left.\frac{1}{2} \frac{\partial \tilde{R}_{\zeta}^{(1)}}{\partial t}\right|_{y=0}
$$

Using the formula

$$
\frac{\partial \tilde{F}_{u}^{(1)}}{\partial t}+\frac{\partial \tilde{F}_{h}^{(1)}}{\partial x}=\frac{\partial^{2} \tilde{v}_{1}}{\partial t^{2}}-\frac{\partial^{2} \tilde{v}_{1}}{\partial x^{2}}+\frac{\partial^{2} \tilde{R}_{v}^{(1)}}{\partial x \partial y}-\frac{\partial \tilde{R}_{v}^{(1)}}{\partial t}+\frac{\partial \tilde{R}_{\varsigma}^{(1)}}{\partial x}-\frac{\partial^{2} \tilde{R}_{\varsigma}^{(1)}}{\partial t \partial y}
$$

and integration by parts we write (5.27) in the form:

$$
\frac{\partial C_{h}^{(1)}}{\partial t}+\frac{\partial C_{h}^{(1)}}{\partial x}=R_{k}=
$$




$$
\begin{aligned}
& \left(\frac{\partial}{\partial t}+\frac{\partial}{\partial x}\right)\left[\frac{1}{2} \int_{0}^{\infty}\left(\frac{\partial \tilde{v}_{1}}{\partial t}-\frac{\partial \tilde{v}_{1}}{\partial x}+\tilde{R}_{v}^{(1)}+\tilde{R}_{\varsigma}^{(1)}\right) e^{-y} d y-\left(\frac{\tilde{u}_{0}^{2}}{2}+\bar{u}_{0} \tilde{u}_{0}\right)_{y=0}\right]-\left.\frac{\partial \tilde{u}_{0}}{\partial T_{1}}\right|_{y=0}+ \\
& \frac{\partial}{\partial t}\left[\left(\frac{\tilde{u}_{0}^{2}}{2}+\bar{u}_{0} \tilde{u}_{0}\right)_{y=0}-\int_{0}^{\infty}\left(\tilde{R}_{v}^{(1)}+\tilde{R}_{\varsigma}^{(1)}\right) e^{-y} d y\right]
\end{aligned}
$$

Equation (5.29) describes the first-order Kelvin waves plus some function bounded in time and space. Analysis of resonant terms in the right hand side $R_{k}$ of (5.29) gives us the equation for slow evolution of the lowest-order Kelvin wave.

\section{Slow evolution of the lowest-order Kelvin wave}

The terms $\frac{\partial}{\partial t}\left(\frac{\tilde{u}_{0}^{2}}{2}\right)_{y=0}$ and $\left.\frac{\partial \tilde{u}_{0}}{\partial T_{1}}\right|_{y=0}$ in $R_{k}$ are definitely resonant, and so the question is if the other terms in $R_{k}$ are resonant. To answer this question the response to various non-linear terms in the right hand side of (5.29) was examined in an analogous way as for the nonlinear source terms in the problem (5.13), (5.14). For the localised initial conditions (2.9c) only the interactions Kelvin wave - Kelvin wave are resonant and the other interactions (Kelvin wave -IG waves, Kelvin wave slow component, IG waves - IG waves, and IG waves - slow component) are not resonant. Therefore the resulting equation for the slow evolution of the lowest-order Kelvin wave in the localised case has the form

$$
\frac{\partial K_{w}^{(0)}}{\partial T_{1}}+K_{w}^{(0)} \frac{\partial K_{w}^{(0)}}{\partial x^{\prime}}=0, \quad x^{\prime}=x-t .
$$

Equation (5.30) should be solved under initial condition (3.34a).

In the periodic and "step" cases the interaction Kelvin wave - slow component turns out to be resonant in addition to the Kelvin wave - Kelvin wave interactions; all other interactions are ineffective. The term in $R_{k}$ related to the Kelvin wave - slow component interaction using (5.1h) and (3.16a) can be written in the form

$$
R_{k m}=\int_{0}^{\infty}\left(\frac{\partial K_{w}^{(0)}}{\partial t} \frac{\partial \bar{v}_{0}}{\partial x}+K_{w}^{(0)} \frac{\partial \Pi_{0}}{\partial x}+\frac{\partial K_{w}^{(0)}}{\partial x} \Pi_{0}\right) e^{-2 y} d y+\left(\bar{u}_{0} \frac{\partial K_{w}^{(0)}}{\partial t}\right)_{y=0} .
$$

Each term in the integrand and in the brackets in the right hand side of (5.31) can be represented as a product

$$
P=a_{k}\left(x, y, t, T_{1}, \ldots\right) b_{s}\left(x, y, T_{1}, \ldots\right)
$$


where $a_{k}$ and $b_{s}$ correspond to the Kelvin wave and slow component, respectively. In the periodic case both these terms are represented as Fourier series in $x$ :

$a_{k}=e^{-y} \sum_{m} a_{k m}\left(T_{1}, \ldots\right) e^{i m(x-t)}, \quad b_{s}=\sum_{n} b_{s n}\left(y, T_{1}, \ldots\right) e^{i n x}$.

and therefore (5.31) takes the form

$R_{k m}=\sum_{m, n} \bar{a}_{k m} \bar{b}_{s n} e^{i(m+n) x-i x t}$

where $\bar{a}_{k m}$ and $\bar{b}_{s n}$ are some coefficients depending on slow times. Obviously, the sum (5.34) is resonant only if the coefficient $\bar{b}_{s 0}$ and therefore $b_{s 0}$ are non-zero, i.e. the slow component includes a zonal current. Simple calculations using $(3.6 \mathrm{a}, \mathrm{b}, \mathrm{c})$ show that the resonant part of $R_{k m}$ can be written as

$R_{k m}^{(r)}=-C\left(T_{1}, \ldots\right) \frac{\partial K_{w}^{(0)}}{\partial x}$

where

$C=-3 \int_{0}^{\infty} \bar{h}_{00} e^{-2 y} d y+2 \bar{h}_{0 B}, \quad \bar{h}_{00}\left(y, T_{1}, \ldots\right)=\left\langle\bar{h}_{0}\right\rangle_{x}, \quad \bar{h}_{0 B}=\left.\bar{h}_{0}\right|_{y=0}$.

The resulting equation for the slow evolution of the lowest order Kelvin wave is written as

$\frac{\partial K_{w}^{(0)}}{\partial T_{1}}+\left[K_{w}^{(0)}+C\left(T_{1}, \ldots\right)\right] \frac{\partial K_{w}^{(0)}}{\partial x^{\prime}}=0$

The initial condition for (5.37) is given by equation (3.32a).

In the "step" case (2.9b) the resonant part of $R_{k m}$ has a form similar to (5.35) but with the coefficient depending on $x$ :

$R_{k m}^{(r)}=-C_{s t}\left(x, T_{1}, \ldots\right) \frac{\partial K_{w}^{(0)}}{\partial x}, \quad C_{s t}=-3 \int_{0}^{\infty} \bar{h}_{0} e^{-2 y} d y+2 \bar{h}_{0 B}$.

The $x$-dependence is related to the integral $F=\int_{0}^{\infty} \bar{h}_{0} e^{-2 y} d y$ which has the form of "step" (see above Sect. 4):

$$
\begin{aligned}
& F=F\left(x, T_{1}, \ldots\right) \rightarrow F^{( \pm)}, \quad x \rightarrow \pm \infty, \\
& F^{( \pm)}=\int_{0}^{\infty} \bar{h}_{0}^{( \pm)}(y) e^{-2 y} d y, \quad \bar{h}_{0}^{( \pm)}=\lim \bar{h}_{0}, x \rightarrow \pm \infty .
\end{aligned}
$$

We demand that the solution be bounded as $t \rightarrow \infty$ in any coordinate frame including one moving with the Kelvin wave phase speed. It follows from this condition that the resonant part $R_{k m}^{(r)}$ takes the form (5.38a) but with constant parameter $C$ equal to 


$$
C=-3 \int_{0}^{\infty} \bar{h}_{0}^{(+)} e^{-2 y} d y+2 \bar{h}_{0 B} .
$$

Correspondingly, the equation for slow evolution of the Kelvin wave in the "step" case has the form (5.37) but with constant $C$ given by (5.41). Note that (5.37) can be reduced to (5.30) by the simple coordinate transformation

$$
x^{\prime} \rightarrow x^{\prime}-X_{C}\left(T_{1}, \ldots\right), \frac{\partial X_{C}}{\partial T_{1}}=C
$$

Thus we see that in all cases the slow evolution of Kelvin waves is governed by the equation for a simple wave (5.30); the presence of mean zonal current in the periodic and "step" cases results only in some Doppler shift of the Kelvin wave phase speed. It is well-known (e.g. Lighthill, 1980) that in general the simple wave breaks in a finite time, and therefore the Kelvin wave behaviour can be characterised as fast propagation of a slowly-breaking profile. Note that the breaking can be prevented if some additional dispersion or friction are incorporated in the model.

\section{Mass conservation in the localised case}

In Section 4 we showed that in the localised case the total mass of the slow component was not conserved, and the first-order slow correction was not localised. Below we will see that the first-order Kelvin wave is also non-localised, and together with the non-localised first-order slow correction compensates the non-conservation of the lowest-order slow field mass.

The first-order correction $\bar{h}_{\boldsymbol{1}}$ obeys the equation following from $(4.4 \mathrm{a}, \mathrm{b}, \mathrm{c})$ :

$$
\nabla^{2} \bar{h}_{1}-\bar{h}_{1}=\Pi_{1}+\operatorname{div} \overline{\mathbf{R}}_{\mathbf{v}}^{(1)}
$$

where $\overline{\mathbf{R}}_{\mathrm{v}}^{(1)}$ is given by (5.5b). To obtain the boundary condition for (5.43) we use equation (4.6) in a slightly modified form,

$$
\left.\frac{\partial \bar{h}_{1}}{\partial x}\right|_{y=0}=\left.\frac{\partial^{2} \bar{h}_{0}}{\partial y \partial T_{1}}\right|_{y=0}-\left.\bar{u}_{0} \frac{\partial \bar{u}_{0}}{\partial x}\right|_{y=0},
$$

one of the conditions $(5.22 \mathrm{a})$,

$$
\left.\tilde{h}_{1}\right|_{y=0}=-\frac{1}{2} \int_{0}^{\infty} \tilde{F}_{h}^{(1)} e^{-y} d y+C_{h}^{(1)},
$$


and the presumed locality of the solution to problem $(2.1 \mathrm{a}, \mathrm{b}),(2.3),(2.5)$ with localised initial conditions $(2.9 \mathrm{c})$.

The solution $C_{h}^{(1)}$ to (5.29) can be represented (since all non-linear terms are localised and tend to zero at $t \rightarrow \infty$ ) as a sum

$$
C_{h}^{(1)}=K_{w}^{(1)}\left(x-t, T_{1}, \ldots\right)+C_{L}(x, t)
$$

where $K_{W}^{(1)}$ is the first-order Kelvin wave amplitude and $C_{L}$ - some localised solution to the inhomogeneous equation (5.27). Since we are looking for a localised solution the sum $h_{1}=\bar{h}_{1}+\tilde{h}_{1}$ should be space-localised. Therefore, using (5.46) and the fact that the function $\tilde{F}_{h}^{(1)}$ is localised, (5.45) can be written as

$\left.\overline{h_{1}}\right|_{y=0}=\bar{h}_{1 B}\left(x, T_{1}, \ldots\right)=-K_{w}^{(1)}\left(x-t, T_{1}, \ldots\right)+F_{L}$

where $F_{L}$ is some localised function. The Kelvin wave $K_{w}^{(1)}\left(x-t, T_{1}, \ldots\right) e^{-y}$ should have zero time average (see (3.28)) therefore from (5.47)

$\bar{h}_{1 B} \rightarrow 0, \quad x \rightarrow-\infty$.

The boundary condition for $\bar{h}$ follows from (5.44), (5.48):

$\left.\bar{h}_{1}\right|_{y=0}=\left.\int_{-\infty}^{x} \frac{\partial^{2} \bar{h}_{0}}{\partial y \partial T_{1}}\right|_{y=0} d x-\left.\frac{\bar{u}_{0}^{2}}{2}\right|_{y=0}$.

The solution $\overline{h_{1}}$ to the problem (5.43), (5.49) can be represented as a sum of non-localised and localised terms, the non-localised term arising when the along wall circulation $\left.\int_{-\infty}^{\infty} \frac{\partial^{2} \bar{h}_{0}}{\partial y \partial T_{1}}\right|_{y=0} d x$ is non-zero. The function $\Pi_{1}$ in (5.43) is localised since by virtue of $(4.4 \mathrm{c}),(5.1 \mathrm{c}) \varsigma_{1}-h_{1}=\Pi_{1}+\tilde{R}_{\varsigma}^{(1)}$ and $\tilde{R}_{\varsigma}^{(1)}$ is localised. Therefore $\bar{h}_{1}$ is represented in the form

$\hbar_{1}=\hbar_{1 B}\left(x, T_{1}, \ldots\right) e^{-y}+\hbar_{11}$,

where $\bar{h}_{11}$ obeys the equations

$\nabla^{2} \bar{h}_{11}-\bar{h}_{11}=\Pi_{1}+\operatorname{div} \overline{\mathbf{R}}_{\mathrm{v}}^{(1)}-\frac{\partial^{2} \bar{h}_{1 B}}{\partial x^{2}} e^{-y},\left.\quad \bar{h}_{11}\right|_{y=0}=0$.

The right-hand side of (5.51a) is localised, and therefore $\bar{h}_{11}$ is also localised.

We now write the elevation $h$ up to $O\left(\varepsilon^{2}\right)$ as,

$$
\begin{aligned}
& h=\bar{h}_{0}+\tilde{h}_{01}+K_{w}^{(0)}\left(x-t, T_{1}, \ldots\right) e^{-y}+ \\
& \varepsilon\left[\bar{h}_{1}+\tilde{h}_{11}+C_{L} e^{-y}+K_{w}^{(1)}\left(x-t, T_{1}, \ldots\right) e^{-y}\right]
\end{aligned}
$$


and integrate (5.52) over the half-plane $y>0$. It readily follows from $(3.17 \mathrm{~b}, \mathrm{c})$ that the "mass" of the fast field $\tilde{h}_{01}$ is conserved (taking into account that $\widetilde{\nabla}_{0}$ in $(3.17 \mathrm{c})$ is odd in $y$ ):

$$
\int_{y>0} \tilde{h}_{01} d x d y=\text { constant }
$$

The same is valid for the lowest-order Kelvin wave solution,

$$
\int_{y>0} K_{w}^{(0)}\left(x-t, T_{1}, \ldots\right) e^{-y} d x d y=\text { constant }
$$

as follows from (5.30). Also the calculations show that the changes of the "mass" of the fast field $\tilde{h}_{1}$ are compensated by the term $C_{L} e^{-y}$, i.e.

$\frac{\partial}{\partial t} \int_{y>0}\left[\tilde{h}_{11}+C_{L} e^{-y}\right] d x d y=0$.

To obtain (5.55) we use equations (5.20b), (5.24), and the equation for $C_{L}$ which coincides with (5.27) where $C_{h}^{(1)}$ is replaced by $C_{L}$.

So, for the total mass to be conserved the term

$S_{0}=\frac{\partial}{\partial T_{1}} \int_{y>0} \bar{h}_{0} d x d y=-\left.\int \frac{\partial^{2} \bar{h}_{0}}{\partial y \partial T_{1}}\right|_{y=0} d x$

should be balanced by the term

$$
S_{1}=\frac{\partial}{\partial t} \int_{y>0}\left[\bar{h}_{1}+K_{w}^{(1)}\left(x-t, T_{1}, \ldots\right) e^{-y}\right] d x d y .
$$

To show that this is really the case we note that by virtue of (5.47), (5.49)

$$
K_{w}^{(1)} \rightarrow-\left.\int \frac{\partial^{2} \bar{h}_{0}}{\partial y \partial T_{1}}\right|_{y=0} d x, \quad x \rightarrow+\infty
$$

whence it follows that the first-order Kelvin wave is not localised, it has a "step like" shape if $\left.\int_{-\infty}^{\infty} \frac{\partial^{2} \bar{h}_{0}}{\partial y \partial T_{1}}\right|_{y=0} d x \neq 0$

Nevertheless the integral in (5.57) converges since the sum $\bar{h}_{1}+K_{w}^{(1)}\left(x-t, T_{1}, \ldots\right) e^{-y}$ is localised as follows from (5.58), (5.50), and (5.47):

$\bar{h}_{1}+K_{w}^{(1)}\left(x-t, T_{1}, \ldots\right) e^{-y}=\left[\bar{h}_{1 B}+K_{w}^{(1)}\left(x-t, T_{1}, \ldots\right)\right] e^{-y}+\bar{h}_{11}$.

Using (5.59), (5.47), and (5.58) one can calculate $S_{1}$ :

$$
S_{1}=\int_{-\infty}^{\infty} \frac{\partial F_{L}}{\partial t} d x=-\int_{-\infty}^{\infty} \frac{\partial K_{w}^{(1)}}{\partial t} d x=-\left.\int_{-\infty}^{\infty} \frac{\partial^{2} \bar{h}_{0}}{\partial y \partial T_{1}}\right|_{y=0} d x .
$$


Thus we see that the terms $S_{0}$ and $S_{1}$ really balance each other and the total mass ("fast" + "slow") is conserved. The sum $\bar{h}_{1}+K_{w}^{(1)}\left(x-t, T_{1}, \ldots\right) e^{-y}$ looks like an injected jet bringing out the surplus or shortage (depending on the sign of $\left.\int_{-\infty}^{\infty} \frac{\partial^{2} \bar{h}_{0}}{\partial y \partial T_{1}}\right|_{y=0} d x$ ) of the mass from the localised lowest-order disturbance.

\section{Initial slow field $\bar{h}_{1}$}

The initial slow field $\bar{h}_{\text {/ }}$ obeys the equation (see (5.43)):

$\nabla^{2} \bar{h}_{1 I}-\bar{h}_{1 I}=\Pi_{1 I}+\operatorname{div} \overline{\mathbf{R}}_{\mathrm{v} I}^{(1)}$.

To obtain the boundary condition for (5.61) at $y=0$ we use equation (5.45) analogous to (3.20a).

Since $\tilde{h}_{1 I}=-\bar{h}_{1 I}$ we have from $(5.45)$ :

$\left.\bar{h}_{1 I}\right|_{y=0}=\frac{1}{2} \int_{0}^{\infty} \tilde{F}_{h I}^{(1)} e^{-y} d y-K_{w}^{(1)}(x)-C_{h f l}^{(1)}$,

where the function $\tilde{F}_{h I}^{(1)}$ (see $\left.(5.18 \mathrm{~b})\right)$ is known because $\tilde{v}_{1 I}$ and $\left.\frac{\partial \tilde{v}_{1}}{\partial t}\right|_{t=0}$ are given by (5.10), (5.11). The function $C_{h}^{(1)}$ in (5.45) is replaced by the sum $C_{h}^{(1)}=K_{w}^{(1)}\left(x-t, T_{1}, \ldots\right)+C_{h f}^{(1)}$ where $K_{w}^{(1)}$ is the amplitude of the first-order Kelvin wave, and $C_{h f}^{(1)}$ is a known particular solution to the inhomogeneous equation (5.27). Again, applying the condition (3.28) to $K_{w}^{(1)}$ one can determine either $\left.\bar{h}_{1 I}\right|_{x=-\infty, y=0}$ in the step case or $\left\langle\left.\bar{h}_{1 I}\right|_{y=0}\right\rangle_{x}$ in the periodic case, and then $\left.\bar{h}_{1 I}\right|_{y=0}$ from equation (4.6) taken at the initial moment. Given $\left.\bar{h}_{1 I}\right|_{y=0}$ we can find from (5.62) the initial profile $K_{w}^{(1)}(x)$ of the first-order Kelvin wave, and the field $\bar{h}_{1 I}$ from (5.61).

Thus the first-order splitting (4.2) is self-consistent for all initial conditions (2.9). The algorithm in use provides the evolution equation for the lowest-order slow field and the Kelvin waves, and a fast correction to the lowest-order fast component. The evolution of the first correction to the slow field is determined at the third order of the perturbation theory (cf. RZB).

\section{Modified QG equation}

The equation for the first-order slow motion is obtained in a manner similar to the method used in Section 3.3 of RZB. We write the third-order vorticity equation: 


$$
\begin{aligned}
& \frac{\partial\left(\varsigma_{2}-h_{2}\right)}{\partial t}+\frac{\partial\left(\varsigma_{1}-h_{1}\right)}{\partial T_{1}}+\frac{\partial \Pi_{0}}{\partial T_{2}}+\frac{\partial\left[u_{0}\left(\varsigma_{1}-h_{1}\right)\right]}{\partial x}+\frac{\partial\left[v_{0}\left(\varsigma_{1}-h_{1}\right)\right]}{\partial y}+ \\
& \frac{\partial\left(u_{1} \Pi_{0}\right)}{\partial x}+\frac{\partial\left(v_{1} \Pi_{0}\right)}{\partial y}=0
\end{aligned}
$$

and average it in time applying (3.5). Using representations (3.3), (4.2) and properties of the fast fields described in Sections 3, 5 one arrives at the equation

$$
\begin{aligned}
& \frac{\partial \Pi_{1}}{\partial T_{1}}+\frac{\partial \Pi_{0}}{\partial T_{2}}+\frac{\partial\left(\bar{u}_{0} \Pi_{1}\right)}{\partial x}+\frac{\partial\left(\bar{v}_{0} \Pi_{1}\right)}{\partial y}+\frac{\partial\left(\bar{u}_{1} \Pi_{0}\right)}{\partial x}+\frac{\partial\left(\bar{v}_{1} \Pi_{0}\right)}{\partial y} \\
& +\frac{\partial\left\langle\tilde{u}_{0}\left(\tilde{\varsigma}_{1}-\tilde{h}_{1}\right)\right\rangle_{t}}{\partial x}=0 .
\end{aligned}
$$

By virtue of $(5.1 \mathrm{c}, \mathrm{g})$ the average $\left\langle\widetilde{u}_{0}\left(\widetilde{\zeta}_{1}-\tilde{h}_{1}\right)\right\rangle_{t}$ is represented as

$$
\left\langle\tilde{u}_{0}\left(\tilde{\zeta}_{1}-\tilde{h}_{1}\right)\right\rangle_{t}=\left\langle\tilde{u}_{0} \tilde{R}_{\varsigma}^{(1)}\right\rangle_{t}=-\left\langle\tilde{u}_{0} \frac{\partial \tilde{U}_{0}}{\partial x}\right\rangle_{t} \Pi_{0} .
$$

Since the fields $\tilde{u}_{01}, \tilde{U}_{01}$ in (3.16a), (5.4a) consisting of IG waves, decaying with increasing time, equation (6.3) is reduced to the equation (see (3.16a), (3.23), and (5.4a)):

$$
\left\langle\widetilde{u}_{0}\left(\widetilde{\zeta}_{1}-\widetilde{h}_{1}\right)\right\rangle_{t}=C_{K} e^{-2 y} \Pi_{0}, C_{K}=\left\langle\left[K_{w}^{(0)}\left(x-t, T_{1}, \ldots\right)\right]^{2}\right\rangle_{t}
$$

The coefficient $C_{K}$ is zero for the "step" and localised initial conditions because in these cases the Kelvin wave amplitude $K_{w}^{(0)}\left(x-t, T_{1}, \ldots\right)$ tends to zero as $x \rightarrow-\infty$ (see (3.28)). But in the periodic case the averaging (3.5) gives:

$$
C_{K}=\frac{1}{2 \pi} \int_{0}^{2 \pi}\left[K_{w}^{(0)}\left(z, T_{1}, \ldots\right)\right]^{2} d z .
$$

As readily follows from (5.37) the coefficient $C_{K}$ is conserved in time (at least, for the time $T_{1}$ ), and therefore it can be calculated directly from the initial Kelvin profile (3.32a).

In order to get a closed equation for $\bar{h}_{0}, \bar{h}_{1}$ we use $(4.4 \mathrm{a}, \mathrm{b}, \mathrm{e}, \mathrm{f})$ to express $\Pi_{1}$ as

$$
\Pi_{1}=\frac{\partial \bar{v}_{1}}{\partial x}-\frac{\partial \bar{u}_{1}}{\partial y}-\bar{h}_{1}=\nabla^{2} \bar{h}_{1}-\bar{h}_{1}-2 J\left(\frac{\partial \bar{h}_{0}}{\partial x}, \frac{\partial \bar{h}_{0}}{\partial y}\right) .
$$

With the help of (4.4a,b,e,f) and the evolution equation (4.3) for $\Pi_{0}=\nabla^{2} \bar{h}_{0}-\bar{h}_{0}$ equation (6.2) takes the following form: 


$$
\begin{aligned}
& \frac{\partial \Pi_{0}}{\partial T_{2}}+\frac{\partial}{\partial T_{1}}\left(\Pi_{1}-\nabla \bar{h}_{0} \nabla \Pi_{0}-\bar{h}_{0} \Pi_{0}\right)+J\left(\bar{h}_{0}, \Pi_{1}-\nabla \bar{h}_{0} \nabla \Pi_{0}-\bar{h}_{0} \Pi_{0}\right)+ \\
& J\left(\bar{h}_{1}-\frac{\left(\nabla \bar{h}_{0}\right)^{2}}{2}+\frac{C_{K}}{2} e^{-2 y}, \Pi_{0}\right)=0 .
\end{aligned}
$$

Equation (6.7) describes a next-order correction to equation (4.3), which is necessary to take into account the slow evolution of the balanced component of the flow for times much longer than $T_{1}$. One can combine the two equations by introducing a "full" slow elevation $\bar{h}=\bar{h}_{0}+\varepsilon \bar{h}_{1}$ (cf. RZB). The resulting equation (which will be referred to as modified quasigeostrophic potential vorticity (MQGPV) equation) can be written in the form of conservation of potential vorticity $\Pi_{M}$ :

$$
\begin{aligned}
& \frac{\partial \Pi_{M}}{\partial T_{1}}+J\left(\bar{h}-\varepsilon \frac{(\nabla \bar{h})^{2}}{2}+\varepsilon \frac{C_{K}}{2} e^{-2 y}, \Pi_{M}\right)=0, \\
& \Pi_{M}=\nabla^{2} \bar{h}-\bar{h}-\varepsilon\left[\bar{h}\left(\nabla^{2} \bar{h}-\bar{h}\right)+\nabla \bar{h} \nabla\left(\nabla^{2} \bar{h}-\bar{h}\right)+2 J\left(\frac{\partial \bar{h}}{\partial x}, \frac{\partial \bar{h}}{\partial y}\right)\right] .
\end{aligned}
$$

Equation (6.8) is constructed in such a way that if the solution $\bar{h}$ is represented as an asymptotic multiple time-scales series,

$\bar{h}=\bar{h}_{0}\left(x, y, T_{1}, \ldots\right)+\varepsilon \bar{h}_{1}\left(x, y, T_{1}, \ldots\right)+\ldots$

then the equations for the corrections $\bar{h}_{0}, \bar{h}_{1}$ coincide with (4.3) and (6.7), respectively. Obviously, (6.8) is valid up to terms $O\left(\varepsilon^{2}\right)$.

In the "step" and localised cases the coefficient $C_{K}$ is zero, and MQGPV equation (6.8) coincides with the "improved" QGPV equation derived in RZB. At the same time, in the periodic case $C_{K} \neq 0$ and the Kelvin wave makes a direct contribution to the slow dynamics, the contribution depending on the initial conditions (2.2). This means, generally, that in the presence of boundary MQGPV equation (6.8) cannot be correctly obtained by a direct expansion of (2.1a,b), (2.5) in $\varepsilon$ assuming all variables to depend on slow times only, as it takes place in the case of an unbounded region (cf. RZB).

The initial conditions for (6.8) are determined by the problem (3.35), (3.29) for $\bar{h}_{0 I}$ and equation (5.61) with the corresponding boundary condition for $\bar{h}_{1 I}$ (see above end of Section 5). The boundary conditions for (6.8) follow from (4.5), (4.6), and from the condition analogous to (4.6) for the second-order slow field $\bar{h}_{2}$,

$$
\left.\frac{\partial \bar{h}_{2}}{\partial x}\right|_{y=0}=-\left(\frac{\partial \bar{u}_{1}}{\partial T_{1}}+\frac{\partial \bar{u}_{0}}{\partial T_{2}}+\frac{\partial}{\partial x}\left(\bar{u}_{0} \bar{u}_{1}\right)\right)_{y=0},
$$

which is derived in the same way as (4.6). In the periodic and "step" cases we have from (4.6):

$$
\left.\bar{h}_{1}\right|_{y=0}=\left.\int \frac{\partial^{2} \bar{h}_{0}}{\partial y \partial T_{1}}\right|_{y=0} d x-\left.\frac{\bar{u}_{0}^{2}}{2}\right|_{y=0}+\bar{h}_{1 B}\left(T_{1}, \ldots\right),
$$


where the first term in the right hand side of (6.11) is the anti-derivative of $\left.\frac{\partial^{2} \bar{h}_{0}}{\partial y \partial T_{1}}\right|_{y=0}$ and $\bar{h}_{1 B}$ is obtained from the condition of boundedness of $\bar{h}_{2}$ using (6.10) and (4.4b):

$$
\left\langle\left(\frac{\partial \bar{u}_{1}}{\partial T_{1}}+\frac{\partial \bar{u}_{0}}{\partial T_{2}}\right)_{y=0}\right\rangle_{x}=-\left\langle\left(\frac{\partial^{2} \bar{h}_{1}}{\partial y \partial T_{1}}+\frac{\partial^{2} \bar{h}_{0}}{\partial y \partial T_{2}}\right)_{y=0}\right\rangle_{x}=0
$$

Combining (4.5) with (6.11), and (4.7) with (6.12) we obtain the boundary

$$
\begin{aligned}
& {\left[\bar{h}-\varepsilon \int \frac{\partial^{2} \bar{h}}{\partial y \partial T_{1}} d x+\frac{\varepsilon}{2}\left(\frac{\partial \bar{h}}{\partial y}\right)^{2}\right]_{y=0}=\bar{h}_{B}\left(T_{1}, \ldots\right),} \\
& \left\langle\left.\frac{\partial^{2} \bar{h}}{\partial y \partial T_{1}}\right|_{y=0}\right\rangle_{x}=0 .
\end{aligned}
$$

Here (6.13b) serves to determine the unknown function $\bar{h}_{B}$ in (6.13a). In the localised case (6.13b) is satisfied identically and $\bar{h}_{B}=0$ in (6.13a).

The MQGPV equation (6.8) together with the initial condition $\bar{h}_{I}=\bar{h}_{0 I}+\varepsilon \bar{h}_{1 I}$ and the boundary conditions (6.13) allows us to determine the evolution of slow component up to nondimensional times $O\left(\varepsilon^{-2}\right)$.

\section{Summary and conclusion}

We have described the process of non-linear geostrophic adjustment in the barotropic RSW model on a half-plane bounded by a rigid wall, applying the multiple time-scale perturbation theory developed in RZB and based on the assumption of a small Rossby number. Different initial states (all localised in $y$-perpendicular to the wall - direction) were considered: periodic in $x$, "step"-like (tending to along-wall rectilinear flows as $x \rightarrow \pm \infty$ ), and localised ones.

Generally, the considered geostrophic adjustment is similar to that in the unbounded plane examined in RZB. In all cases the initial perturbation is split in a unique way into slow and fast components evolving with characteristic time-scales $f^{-1}$ and $(\varepsilon f)^{-1}$, respectively. The slow component is not influenced by the fast one, at least for times $t \leq(\varepsilon f)^{-1}$, and remains close to geostrophic balance. The fast component consists mainly of linear IG waves rapidly propagating outward the initial disturbance and Kelvin waves confined near the boundary. Like the unbounded case considered in RZB, the nonlinear interactions of IG waves with each other, with slow component and the Kelvin waves result only in a small correction to the fast field. 
At the same time, the presence of the boundary brings in a number of new effects into the geostrophic adjustment process. First of all, Kelvin waves arise which propagate along the boundary in such a way that the boundary is on the right in the direction of propagation. The theory provides simple formulae allowing us to construct the initial profile of the Kelvin wave given arbitrary initial conditions. The lowest order fields of the theory describe the process of linear geostrophic adjustment of an arbitrary initial state on the bounded half-plane. With increasing time the Kelvin wave profile slowly distorts, the distortion being due to the Kelvin wave non-linear selfinteraction and being described by the simple-wave equation. The resulting evolution of the initial Kelvin wave profile can be characterised as fast propagation, with slow breaking.

Probably, the most important results of this work is that the presence of Kelvin waves does not prevent from the fast-slow splitting in spite of the fact that (unlike IG waves; see RZB) the frequency gap between the Kelvin waves and slow motion is absent (i.e. Kelvin waves with arbitrarily small frequencies can exist). The possibility of splitting is explained by the special structure of the Kelvin waves in each case considered here. For the periodic initial conditions the Kelvin wave profile is also periodic but it has zero mean which physically corresponds to the absence of a zero-frequency harmonic in the Kelvin wave spectrum. For the localised and "step"like initial conditions such harmonics can be present in the Kelvin wave spectrum, but the shape of the Kelvin wave profile is such that the Kelvin wave field rapidly decays at a fixed spatial point when the Kelvin wave propagates off this point.

Evolution of the slow motion on times $\quad t \leq(\varepsilon f)^{-1}$ is governed by the well-known quasigeostrophic potential vorticity equation for the elevation $\bar{h}_{0}$. The theory provides an algorithm to determine the initial slow and fast fields to any order in $\varepsilon$. The boundary condition used in this non-viscous model is the no flux condition on the rigid wall. Being formulated in terms of $\bar{h}_{0}$, this condition contains some arbitrary function $\bar{h}_{0 B}\left(T_{1}, \ldots\right)$ which is constant along the boundary but depends on the slow time (e.g. Kamenkovich \& Reznik 1978, Pedlosky 1987).

To remove this uncertainty the conservation of along-boundary circulation is used (e.g. Pedlosky 1987). If the fast component is absent then the slow circulation is assumed to be conserved, this conservation providing the energy and mass conservation of the lowest-order slow motion. However, if the initial conditions are not balanced, so that the fast component is present, then the total circulation (fast + slow) should be conserved. The question is if the fast and slow circulations are conserved separately. Our analysis demonstrates that this is really the case for the periodic and "step" like initial conditions.

The situation for localised initial conditions is somewhat more complicated. The lowest-order slow motion in this case is also localised and the elevation $\bar{h}_{0}$ is zero at the boundary, i.e. the 
problem for $\bar{h}_{0}$ is well-defined without using the conservation of slow circulation. Moreover, a simple analysis shows that, generally, the lowest-order circulation and, therefore, the total mass of slow localised motion are not conserved. Conservation of the total circulation and mass is provided by the first-order slow correction $\bar{h}_{1}$ and Kelvin wave $K_{w}^{(1)} e^{-y}$. Separately, neither $\bar{h}_{1}$, nor the Kelvin wave amplitude $K_{w}^{(1)}$, are localised in $x$ but the sum $\bar{h}_{1}+K_{w}^{(1)} e^{-y}$ is localised and looks like an injected jet bringing out the surplus, or shortage of mass from the localised lowest-order slow disturbance.

Note that Dorofeyev \& Larichev (1992) met an analogous problem when considering the reflection of linear Rossby waves from the meridional boundary in the framework of a shallow water model on the $\beta$-plane. They revealed that the total mass of Rossby waves is not conserved, and the surplus or shortage of mass is brought out by fast Kelvin waves.

On longer times $t \leq\left(\varepsilon^{2} f\right)^{-1}$ the slow motion obeys the so-called modified quasigeostrophic potential vorticity (MQGPV) equation. The theory provides initial and boundary conditions for this equation. The MQGPV equation exactly coincides with the "improved" QGPV equation, derived in RZB, in the "step" like and localised cases. In the periodic case this equation contains an additional term due to the Kelvin wave self-interaction, this term depending on the initial Kelvin wave profile. This means, generally, that in the presence of a boundary the MQGPV equation cannot be correctly obtained by a direct expansion of the RSW system in $\varepsilon$ assuming all variables to depend on slow times only, as it takes place in the case of unbounded region.

This work was supported by RFBR Grant 99-05-64841. G.M.R. gratefully acknowledges the hospitality of Loughborough University where the main part of the work was done.

\section{References}

Babin, A., Mahalov, A., and Nikolaenko, B., 1998a, "Global splitting and regularity of rotating shallow-water equations", Europ. Journ. of Mech. B/Fluids, 15(3), p. 291-300.

Babin, A., Mahalov, A., and Nikolaenko, B., 1998b, "Regularity and Integrability of rotating shallow-water equations", C. R. Acad. Sci. Paris Ser. I, 324, p. 593-598.

Blumen, W., 1972, “Geostrophic adjustment”, Rev. Geophys. And Space Phys., 10, p. 485-528.

Dewar, W.K., Killworth, P.D., 1995, “Do fast gravity waves interact with geostrophic motions?”, Deep-Sea Research I, 42, p. 1063-1081. 
Dorofeyev , V.L., Larichev, V.D., 1992, "The exchange of fluid mass between quasi-geostrophic and ageostrophic motions during the reflection of Rossby waves from a coast. 1 . The case of an infinite rectilinear coast", Dyn. Atmos. Oceans, 16(3-4), p. 305-329.

Gill, A.E., 1976, “Adjustment under gravity in a rotating channel”, J. Fluid Mech., 77, p. 603-621.

Lighthill, J., 1980, “Waves in fluids”, Cambridge Univ. Press., 504 p.

Kamenkovich, V.M., Reznik, G.M., 1978, "Rossby waves". In: Physics of the Ocean. V.2. Hydrodynamics of the Ocean, Moscow, Nauka, 1978, p. 300-358, (in Russian)

Medvedev, S.B., 1997, "The slow manifold for the shallow water equations on the $f$-plane", $J$. Atmos. Sci., 56, p. 1050-1054.

Monin, A.S., Obukhov, A.M., 1958, "Small oscillations of the atmosphere and adjustment of meteorological fields", Izvestia - Geophysics, 11, p. 1360-1373 (in Russian).

Obukhov, A.M., 1949, "On the problem of geostrophic wind”, Izvestia - Geography and Geophysics, 13, p. 281-306 (in Russian).

Olver, F.W.J., 1974, "Introduction to asymptotic and special functions", AP, NY, Ch.3.

Pedlosky, J., 1987, “Geophysical fluid dynamics”, Springer, NY.

Reznik, G.M., Zeitlin, V., and Ben Jelloul, M., 2001, "Nonlinear theory of geostrophic adjustment. Part 1. Rotating shallow-water model", J. Fluid. Mech., 445, p. 93-120.

Rossby, C.-G., 1938, “On the mutual adjustment of pressure and velocity distributions in certain simple current systems”, J. Marine Res., 1, p. 239-263.

Warn, T., Bokhove, O., Shepherd, T.G., and Vallis, G.K., 1995, "Rossby number expansions, slaving principles, and balanced dynamics”, Q. J. R. Meteorol. Soc., 121, p. 723-739. 


\section{Appendix A}

In the periodic case (2.9a) the initial fields $F^{o d}, G^{o d}$ are periodic in $x$ and can be represented as

$\left(F^{o d}, G^{o d}\right)=\sum_{m=-\infty}^{m=\infty}\left(f_{m}^{o d}(y), g_{m}^{o d}(y)\right) e^{i m x}$.

where $f_{m}^{O d}, g_{m}^{O d}$ are also odd functions. The solution to the problem $(3.11),(3.13),(\mathrm{A} .1)$ is represented in the form

$$
\tilde{v}_{0 f}=\sum_{m=-\infty}^{m=\infty} \tilde{v}_{0 m}(y, t) e^{i m x}
$$

Substitution of (A.2) into (3.11), (3.13) gives

$-\frac{\partial^{2} \tilde{v}_{0 m}}{\partial t^{2}}+\frac{\partial^{2} \tilde{v}_{0 m}}{\partial y^{2}}-\left(m^{2}+1\right) \tilde{v}_{0 m}=0, \quad\left(\tilde{v}_{0 m}, \frac{\partial \tilde{v}_{0 m}}{\partial t}\right)_{t=0}=\left(f_{m}^{o d}, g_{m}^{o d}\right)$.

The system (A.3) is solved using the Fourier transformation:

$$
\tilde{v}_{0 m}=\int_{-\infty}^{\infty} \hat{v}_{0 m}(l, t) e^{i l y} d l, \quad \hat{v}_{0 m}=A_{m}^{(+)} e^{i \omega_{m l} t}+A_{m}^{(-)} e^{-i \omega_{m l} t}
$$

where $\omega_{m l}$ is the frequency of IG waves,

$\omega_{m l}=\sqrt{m^{2}+l^{2}+1}$

and

$$
A_{m}^{( \pm)}=\frac{1}{2}\left(\hat{f}_{m} \mp \frac{i \hat{g}_{m}}{\omega_{m l}}\right), \quad\left(f_{m}^{o d}, g_{m}^{o d}\right)=\int_{-\infty}^{\infty}\left(\hat{f}_{m}(l), \hat{g}_{m}(l)\right) e^{i l y} d l .
$$

It is of importance for the following estimates that $A_{m}^{( \pm)}(l)$ is an odd function of the wavenumber $l$, i.e.

$A_{m}^{( \pm)}(l)=O(l), \quad l \rightarrow 0$.

The formulae (A.2), (A.4) to (A.6) give the solution for $\tilde{v}_{0 f}$. The function $\tilde{v}_{00}$ has the form analogous (A.2), (A.4) but with unknown amplitudes $A_{m}^{( \pm)}$depending on the slow times $T_{n}$ and equal to zero for $T_{1}=T_{2}=\ldots 0$.

In the "step" case the solution $\tilde{v}_{0 f}$ is, generally, neither periodic nor localised in $x$ unless the initial zonal motion at $y= \pm \infty$ is geostrophic. Therefore to represent $\tilde{v}_{0 f}$ as a superposition of linear waves we find the Fourier representation for the localised derivative $\frac{\partial \tilde{v}_{0 f}}{\partial x}$, and also for the limiting 
fields $\tilde{v}_{0 f}^{( \pm)}(y, t)\left(\tilde{v}_{0 f} \rightarrow \tilde{v}_{0 f}^{( \pm)}(y, t), \quad y \rightarrow \pm \infty\right)$ to compose the desired solution for $\tilde{v}_{0 f}$. The problem for $\frac{\partial \tilde{v}_{0 f}}{\partial x}$ is written as

$L_{w}\left(\frac{\partial \tilde{v}_{0 f}}{\partial x}\right)=0,\left.\quad \frac{\partial \tilde{v}_{0 f}}{\partial x}\right|_{y=0}=0, \quad\left(\frac{\partial \tilde{v}_{0 f}}{\partial x}, \frac{\partial^{2} \tilde{v}_{0 f}}{\partial x \partial t}\right)_{t=0}=\left(\frac{\partial F}{\partial x}, \frac{\partial G}{\partial x}\right)$

where $L_{w}$ is the linear operator in (3.11). The solution to (A.8) is readily written in the form of two-dimensional Fourier integrals (see, e.g. RZB),

$$
\frac{\partial \tilde{v}_{0 f}}{\partial x}=\int_{-\infty}^{\infty} \hat{v}_{0 x}(k, l, t) e^{i(k x+l y)} d k d l, \quad \hat{v}_{0 x}=A^{(+)} e^{i \omega_{k l} t}+A^{(-)} e^{-i \omega_{k l} t}
$$

where

$\omega_{k l}=\sqrt{k^{2}+l^{2}+1}, \quad A^{( \pm)}=\frac{1}{2}\left(\hat{F}_{x}(k, l) \mp \frac{i \hat{G}_{x}(k, l)}{\omega_{k l}}\right)$,

and the functions $\hat{F}_{x}, \hat{G}_{x}$ are Fourier transformations of the functions $F^{o d}, G^{o d}$. Obviously, $\hat{F}_{x}, \hat{G}_{x}$, and, therefore, $A^{( \pm)}$are also odd functions in $l$,

$A^{( \pm)}=O(l), \quad l \rightarrow 0$.

The system for $\tilde{v}_{0 f}^{( \pm)}(y, t)$ coincides with (A.3) if we put $m=0, f_{m}^{o d}=0$ (the function $F$ is localised), and $g_{m}^{\text {od }}=G^{( \pm)}(y)$ where

$G^{( \pm)}(y)=\lim G^{o d}(x, y), x \rightarrow \pm \infty$.

The solution $\widetilde{v}_{0 f}^{( \pm)}(y, t)$ has the form

$$
\tilde{v}_{0 f}^{( \pm)}=\int_{-\infty}^{\infty} \hat{v}_{0}^{( \pm)}(l, t) e^{i l y} d l, \quad \hat{v}_{0}^{( \pm)}=A_{p}^{( \pm)} e^{i \omega_{01} t}+A_{m}^{( \pm)} e^{-i \omega_{01} t}
$$

where

$$
A_{p}^{( \pm)}=-\frac{i \hat{G}^{( \pm)}}{2 \omega_{0 l}}, A_{m}^{( \pm)}=\frac{i \hat{G}^{( \pm)}}{2 \omega_{0 l}}
$$

To find $\widetilde{\nabla}_{0} f$ we use a simple identity

$$
\tilde{v}_{0 f}=\tilde{v}_{01}+\tilde{v}_{0 f}^{( \pm)}-\tilde{v}_{01}^{( \pm)},
$$

where

$$
\tilde{v}_{01}=\int_{0}^{x} \frac{\partial \tilde{v}_{0 f}}{\partial x} d x, \quad \tilde{v}_{01}^{( \pm)}(y, t)=\lim \tilde{v}_{01}, x \rightarrow \pm \infty .
$$

Representing $\tilde{v}_{01}$ in the form 


$$
\tilde{v}_{01}=-\int_{-\infty}^{\infty} \hat{v}_{0 x}(k, l, t) e^{i l y} \frac{1-e^{i k x}}{i k} d k d l
$$

and calculating $\tilde{v}_{01}^{( \pm)}$from (A.17) we arrive at the equation

$$
\tilde{v}_{0 f}=-i \int_{-\infty}^{\infty} d l f \frac{\hat{v}_{0 x}(k, l, t)}{k} e^{i(k x+l y)} d k+\int_{-\infty}^{\infty} \hat{g}_{v}(l, t) e^{i l y} d l,
$$

$\hat{g}_{v}=\hat{v}_{0 f}^{(-)}(l, t)+\pi \hat{v}_{0 x}(0, l, t)$

where $f$ denotes the Cauchy principal value integral over $k$ Equation (A.18) expresses $\widetilde{v}_{0 f}$ as a superposition of plane IG waves. Again, the slow time-dependent part $\tilde{v}_{00}$ has a form analogous to (A.18) but with unknown amplitudes $A^{( \pm)}$in (A.9b) and $\hat{g}_{v}$ depending on slow times and equal to zero at $T_{1}=T_{2}=\ldots 0$.

For the localised case (2.9c) the solution $\tilde{v}_{0 f}$ has the form (A.9), (A.10):

$$
\begin{aligned}
& \tilde{v}_{0 f}=\int_{-\infty}^{\infty} \hat{v}_{0 f}(k, l, t) e^{i(k x+l y)} d k d l, \quad \hat{v}_{0 f}=A^{(+)} e^{i \omega_{k l} t}+A^{(-)} e^{-i \omega_{k l} t}, \\
& A^{( \pm)}=\frac{1}{2}\left(\hat{F}(k, l) \mp \frac{i \hat{G}(k, l)}{\omega_{k l}}\right) .
\end{aligned}
$$

Of course, the property (A.11) for $A^{( \pm)}$holds in this case too.

The fields $\tilde{u}_{01}, \tilde{h}_{01}$ in (3.16) can be also expressed in the form of a superposition of IG waves using the representations (3.17). For the periodic case we have:

$$
\left(\tilde{u}_{01}, \tilde{h}_{01}\right)=\sum_{m=-\infty}^{m=\infty}\left(\tilde{u}_{01}^{(m)}(y, t), \tilde{h}_{01}^{(m)}(y, t)\right) e^{i m x},
$$

where

$$
\begin{aligned}
& \left(\tilde{u}_{01}^{(m)}(y, t), \tilde{h}_{01}^{(m)}(y, t)\right)=\int_{-\infty}^{\infty}\left(\hat{u}_{01}^{(m)}(l, t), \hat{h}_{01}^{(m)}(l, t)\right) e^{i l y} d l \\
& \hat{u}_{01}^{(m)}=\frac{1}{l^{2}+1}\left(m l \hat{v}_{0 m}-\frac{\partial \hat{v}_{0 m}}{\partial t}\right), \quad \hat{h}_{01}^{(m)}=\frac{i}{l^{2}+1}\left(m \hat{v}_{0 m}+l \frac{\partial \hat{v}_{0 m}}{\partial t}\right) .
\end{aligned}
$$

For the "step" case (2.9b)

$$
\begin{aligned}
& \left(\tilde{u}_{01}, \tilde{h}_{01}\right)=-i \int_{-\infty}^{\infty} d l f \frac{\left(\hat{u}_{0 x}, \hat{h}_{0 x}\right)}{k} e^{i(k x+l y)} d k+\int\left(\hat{g}_{u}, \hat{g}_{h}\right) e^{i l y} d l \\
& \hat{u}_{0 x}=\frac{1}{l^{2}+1}\left(k l \hat{v}_{0 x}-\frac{\partial \hat{v}_{0 x}}{\partial t}\right), \quad \hat{h}_{0 x}=\frac{i}{l^{2}+1}\left(k \hat{v}_{0 x}+l \frac{\partial \hat{v}_{0 x}}{\partial t}\right), \\
& \left(\hat{g}_{u}, \hat{g}_{h}\right)=\frac{(-1, i l)}{l^{2}+1} \frac{\partial}{\partial t}\left[\hat{v}_{0 f}^{(-)}(l, t)+\pi \hat{v}_{0 x}(0, l, t)\right] .
\end{aligned}
$$

Finally, in the localised case (2.9c) 


$$
\begin{aligned}
& \left(\tilde{u}_{01}, \tilde{h}_{01}\right)=\int_{-\infty}^{\infty}\left(\hat{u}_{01}, \hat{h}_{01}\right) e^{i(k x+l y)} d k d l \\
& \hat{u}_{01}=\frac{1}{l^{2}+1}\left(k l \hat{v}_{0 f}-\frac{\partial \hat{v}_{0 f}}{\partial t}\right), \quad \hat{h}_{01}=\frac{i}{l^{2}+1}\left(k \hat{v}_{0 f}+l \frac{\partial \hat{v}_{0 f}}{\partial t}\right)
\end{aligned}
$$

\section{Appendix B}

All estimates will be made for the simplest case of localised initial conditions; the generalisation to the periodic and "step" cases can be readily done using the representation of the fast fields in these cases as a superposition of harmonic IG waves and Kelvin waves (see Appendix A).

\section{Estimates for $\tilde{v}_{0 f}, \tilde{u}_{0}, \tilde{h}_{0}$}

Estimation of $\widetilde{v}_{0} f$ in (A.20a) at large times is reduced to the estimation of the integrals,

$$
J^{ \pm \pm}=\int_{-\infty}^{\infty} A^{( \pm)}(k, l) e^{i(k x+l y) \pm \omega_{k l} t} d k d l
$$

where $A^{( \pm)}$is given by (A.21). We will consider $J^{(+)}$; the integral $J^{(-)}$can be estimated in the same way. It is convenient to write (B.1) in the form

$J^{(+)}=\int_{0}^{\infty} d k \int_{0}^{\infty} A_{1}^{(+)}(k, l, x, y) e^{i \omega_{k l} t} d l$,

where

$$
\begin{aligned}
& A_{1}^{(+)}=\left[A^{(+)}(k, l) e^{i l y}+A^{(+)}(k,-l) e^{-i l y}\right] e^{i k x}+ \\
& {\left[A^{(+)}(-k, l) e^{i l y}+A^{(+)}(-k,-l) e^{-i l y}\right] e^{-i k x}}
\end{aligned}
$$

We now estimate the internal integral in (B.2) for $t \rightarrow \infty, x, y$ fixed,

$$
J_{l}=\int_{0}^{\infty} A_{1}^{(+)}(k, l, x, y) e^{i \omega_{k l} t} d l
$$

using that

$A_{1}^{(+)}=O\left(l^{2}\right), \quad l \rightarrow 0$

due to the oddness of $A^{(+)}$in $l$ (see Appendix A). Integrating by parts, taking into account of (B.5) gives,

$J_{l}=\frac{1}{t} \int_{0}^{\infty} A_{2}^{(+)}(k, l, x, y) e^{i \omega_{k l} t} d l$

where 
$A_{2}^{(+)}=i\left(\frac{A_{1}^{(+)}}{\omega_{k l}^{\prime}}\right)^{\prime}=O(1), \quad l \rightarrow 0$.

The prime denotes differentiation with respect to $l$.

The integral in (B.6) is estimated using the standard stationary phase method (e.g. Olver, 1974). The stationary point (corresponding to zero group velocity) here is $l=0$; as a result we have $J_{l}=O\left(\frac{1}{t^{3 / 2}}\right), t \rightarrow \infty, x, y$ fixed.

The final estimate for the integral $J^{( \pm)}$is

$J^{( \pm)}=O\left(\frac{1}{t^{2}}\right), t \rightarrow \infty, x, y$ fixed.

Faster decay of $J^{( \pm)}$in comparison with $J_{l}$ is explained by the additional integrating over $k$ of the integrand containing an exponent $e^{i \omega_{k 0} t}$. In the periodic and "step" cases this integration is absent, and therefore the integral $J^{( \pm)}$behaves like $J_{l}$ in these cases. Analogous estimates for $\tilde{u}_{01}, \tilde{h}_{01}$ are performed in the same way using the representations (A.24), so in all cases the estimates (3.27) hold.

Estimates of the forced solution to the problem (5.13a), (5.14)

Interaction IG wave - IG wave

The typical forcing term $\Phi_{1}^{(i g)} \Phi_{2}^{(i g)}$ can be written as

$\Phi_{1}^{(i g)} \Phi_{2}^{(i g)}=\int_{-\infty}^{\infty} A_{1}\left(k_{1}, l_{1}\right) A_{2}\left(k_{2}, l_{2}\right) e^{i\left[\left(k_{1}+k_{2}\right) x+\left(l_{1}+l_{2}\right) y+\Omega^{( \pm)} t\right]} d k_{12} d l_{12}$

where

$d k_{12}=d k_{1} d k_{2}, \quad d l_{12}=d l_{1} d l_{2}, \quad \Omega^{( \pm)}=\omega_{k_{1} l_{1}} \pm \omega_{k_{2} l_{2}}$

and

$A_{i}\left(k_{i}, l_{i}\right)=O\left(l_{i}\right), l_{i} \rightarrow 0, \quad i=1,2$.

The response $\tilde{v}_{w w}$ to this forcing satisfying the equation

$L_{w} \tilde{v}_{w w}=\Phi_{1}^{(i g)} \Phi_{2}^{(i g)}, \quad L_{w}=-\frac{\partial^{2}}{\partial t^{2}}+\nabla^{2}-1$

can be written as

$\tilde{v}_{w w}=\int_{-\infty}^{\infty} A_{1}\left(k_{1}, l_{1}\right) A_{2}\left(k_{2}, l_{2}\right) e^{i\left[\left(k_{1}+k_{2}\right) x+\left(l_{1}+l_{2}\right) y\right]} \frac{e^{i \Omega^{( \pm)} t}}{\left(\Omega^{( \pm)}\right)^{2}-\omega_{k l}^{2}} d k_{12} d l_{12}$

where $(k, l)=\left(k_{1}+k_{2}, l_{1}+l_{2}\right)$.

Triad interactions of IG waves are impossible therefore the denominator in the integrand in (B.14) never vanishes. Integration by parts over $l_{1}$ and $l_{2}$ in (B.14) using (B.12) gives the following estimate 
$\tilde{v}_{w w}=O\left(\frac{1}{t^{3}}\right), t \rightarrow \infty, x, y$ fixed.

\section{Interaction IG wave - slow motion}

This estimate is the most tedious. The forcing term $\Phi_{0}^{(s)} \Phi_{0}^{(i g)}$ has the form

$$
\Phi_{0}^{(s)} \Phi_{0}^{(i g)}=\int_{-\infty}^{\infty} A_{1}\left(k_{1}, l_{1}\right) A_{2}\left(k_{2}, l_{2}\right) e^{i\left[\left(k_{1}+k_{2}\right) x+\left(l_{1}+l_{2}\right) y \pm \omega_{k 11} t\right]} d k_{12} d l_{12}
$$

where $A_{1}, A_{2}$ satisfy (B.12). It is convenient to introduce the new variables $k_{1}, l_{1}, k=k_{1}+k_{2}$, $l=l_{1}+l_{2}$ and then to reduce the integration over $l, l_{1}$ to integration over $l>0, l_{1}>0$ :

$\Phi_{0}^{(s)} \Phi_{0}^{(i g)}=\int_{-\infty}^{\infty} d k_{01} e^{i k x} \int_{0}^{\infty} F\left(k_{1}, l_{1}, k, l, y\right) e^{ \pm i \omega_{k_{11} t} t} d l_{01}$

where $d k_{01}=d k d k_{1}, d l_{01}=d l d l_{1}$ and

$$
\begin{aligned}
& F=\left[A_{1}\left(k_{1}, l_{1}\right) A_{2}\left(k-k_{1}, l-l_{1}\right)+A_{1}\left(k_{1},-l_{1}\right) A_{2}\left(k-k_{1}, l+l_{1}\right)\right] e^{i l y}+ \\
& {\left[A_{1}\left(k_{1}, l_{1}\right) A_{2}\left(k-k_{1},-l-l_{1}\right)+A_{1}\left(k_{1},-l_{1}\right) A_{2}\left(k-k_{1},-l+l_{1}\right)\right] e^{-i l y}}
\end{aligned}
$$

The integrand in (B.17) contains resonant harmonics therefore the response $\tilde{v}_{s w}$ is represented in the form

$\tilde{v}_{s w}=\int_{-\infty}^{\infty} d k_{01} e^{i k x} \int_{0}^{\infty} F\left(k_{1}, l_{1}, k, l, y\right) \frac{e^{i \omega_{k_{1} l_{1}} t}-e^{i \omega_{k l} t}}{\omega_{k_{1} l_{1}}^{2}-\omega_{k l}^{2}} d l_{01}$.

We have chosen the + sign in the exponent in the integrand of (B.17) for definiteness.

Introducing the new variables $X=\omega_{k l}, Y=\omega_{k_{1} l_{1}}$ instead of $l, l_{1}$,

$l=\sqrt{X^{2}-X_{B}^{2}}, \quad l_{1}=\sqrt{Y^{2}-Y_{B}^{2}}, X_{B}=\sqrt{k^{2}+1}, \quad Y_{B}=\sqrt{k_{1}^{2}+1}$

one obtains

$\tilde{v}_{s w}=\int d k_{01} e^{i k x} f_{h}\left(k_{1}, k, t\right)$

where

$f_{h}\left(k_{1}, k, t\right)=\int_{X_{B}}^{\infty} d X \int_{Y_{B}}^{\infty} \frac{F\left(k_{1}, l_{1}, k, l, y\right) X Y}{\sqrt{X^{2}-X_{B}^{2}} \sqrt{Y^{2}-Y_{B}^{2}}(X+Y)} \frac{e^{i Y t}-e^{i X t}}{Y-X} d Y$.

For definiteness we put

$X_{B}>Y_{B}$

and represent $f_{h}$ in the form

$f_{h}=f_{h}^{(1)}+f_{h}^{(2)}+f_{h}^{(3)}+f_{h}^{(4)}+f_{R}$

where 


$$
\begin{aligned}
& f_{h}^{(1)}=\int_{X_{B}}^{X_{B}+\varepsilon} d X \int_{Y_{B}}^{X_{B}-\varepsilon}() d Y, \quad f_{h}^{(2)}=\int_{X_{B}}^{X_{B}+\varepsilon} d X \int_{X+\varepsilon}^{\infty}() d Y, \\
& f_{h}^{(3)}=\int_{X_{B}}^{X_{B}+\varepsilon} d X \int_{X_{B}-\varepsilon}^{X+\varepsilon}() d Y, \quad f_{h}^{(4)}=\int_{X_{B}+\varepsilon}^{\infty} d X \int_{X-\varepsilon}^{X+\varepsilon}() d Y .
\end{aligned}
$$

The term $f_{R}$ in (B.24a) corresponds to the integration over "non-resonant" domain outside the vicinity of the resonance line $Y=X$ and of the point $X=X_{B}$ (correponding to zero group velocity). Simple estimate shows that

$$
f_{R}=O\left(\frac{1}{t}\right), t \rightarrow \infty \text {. }
$$

The "resonant" term $f_{h}^{(4)}$ can be represented in the form

$f_{h}^{(4)} \cong \int_{X_{B}+\varepsilon}^{\infty} \frac{F\left(k_{1}, l_{1}, k, l, y\right)_{X=Y} X e^{i X t}}{2 \sqrt{X^{2}-X_{B}^{2}} \sqrt{X^{2}-Y_{B}^{2}}} d X \int_{X-\varepsilon}^{X+\varepsilon} \frac{e^{i(Y-X) t}-1}{Y-X} d Y$.

We have for the internal integral:

$J_{1}=\int_{X-\varepsilon}^{X+\varepsilon} \frac{e^{i(Y-X) t}-1}{Y-X} d Y=\int_{-\varepsilon}^{\varepsilon} \frac{e^{i u t}-1}{u} d u=i \pi+O\left(\frac{1}{t}\right), t \rightarrow \infty$,

therefore integration by parts over $X$ in (B.26) gives:

$f_{h}^{(4)}=O\left(\frac{1}{t}\right), t \rightarrow \infty$.

We now write the term $f_{h}^{(3)}$ as

$$
f_{h}^{(3)} \cong \frac{F\left(k_{1}, l_{1}, k, l, y\right)_{X=Y=X_{B}} \sqrt{X_{B}}}{2 \sqrt{2} \sqrt{X_{B}^{2}-Y_{B}^{2}}} \int_{X_{B}}^{X_{B}+\varepsilon} d X \int_{X_{B}-\varepsilon}^{X+\varepsilon} \frac{e^{i Y t}-e^{i X t}}{(Y-X) \sqrt{X-X_{B}}} d Y
$$

and estimate the integral term

$$
J_{2}=\int_{X_{B}}^{X_{B}+\varepsilon} d X \int_{X_{B}-\varepsilon}^{X+\varepsilon} \frac{e^{i Y t}-e^{i X t}}{(Y-X) \sqrt{X-X_{B}}} d Y=e^{i X_{B} t} \int_{0}^{\varepsilon} \frac{e^{i u t}}{\sqrt{u}} d u \int_{-\varepsilon}^{u+\varepsilon} \frac{e^{i(v-u) t}-1}{v-u} d u .
$$

for $t \rightarrow \infty$. Resulting estimate for $f_{h}^{(3)}$ is written as:

$$
f_{h}^{(3)}=\frac{e^{i X_{B} t}}{\sqrt{t}}\left\{i \pi C_{0}\left[\frac{F\left(k_{1}, l_{1}, k, l, y\right) \sqrt{X_{B}}}{2 \sqrt{2} \sqrt{X_{B}^{2}-Y_{B}^{2}}}\right]_{X=Y=X_{B}}+O\left(\frac{\ln t}{\sqrt{t}}\right)\right\}
$$

where

$$
C_{0}=\int_{0}^{\infty} \frac{e^{i z}}{\sqrt{z}} d z
$$

The term $f_{h}^{(1)}$ in (B.24a) is estimated in a similar way:

$f_{h}^{(1)}=f_{h}^{(11)}-f_{h}^{(12)}$, 
$f_{h}^{(11)}=\int_{X_{B}}^{X_{B}+\varepsilon} d X \int_{Y_{B}}^{X_{B}-\varepsilon} \frac{F\left(k_{1}, l_{1}, k, l, y\right) X Y}{\sqrt{X^{2}-X_{B}^{2}} \sqrt{Y^{2}-Y_{B}^{2}}(X+Y)} \frac{e^{i Y t}}{Y-X} d Y=O\left(\frac{1}{t}\right)$,

$f_{h}^{(12)}=\int_{X_{B}}^{X_{B}+\varepsilon} d X \int_{Y_{B}}^{X_{B}-\varepsilon} \frac{F\left(k_{1}, l_{1}, k, l, y\right) X Y}{\sqrt{X^{2}-X_{B}^{2}} \sqrt{Y^{2}-Y_{B}^{2}}(X+Y)} \frac{e^{i Y t}}{Y-X} d Y \cong$

$\sqrt{\frac{X_{B}}{2}} \int_{X_{B}}^{X_{B}+\varepsilon} \frac{e^{i X t} d X}{\sqrt{X-X_{B}}} \int_{Y_{B}}^{X_{B}-\varepsilon} \frac{F\left(k_{1}, l_{1}, k, l, y\right) Y}{\left(Y^{2}-X_{B}^{2}\right) \sqrt{Y^{2}-Y_{B}^{2}}} d Y=$

$\left[\sqrt{\frac{X_{B}}{2}} \int_{Y_{B}}^{X_{B}-\varepsilon} \frac{F\left(k_{1}, l_{1}, k, l, y\right)_{X=X_{B}} Y}{\left(Y^{2}-X_{B}^{2}\right) \sqrt{Y^{2}-Y_{B}^{2}}} d Y\right] C_{0} \frac{e^{i X_{B} t}}{\sqrt{t}}+O\left(\frac{1}{t}\right)$

The resulting estimate for $f_{h}^{(1)}$ takes the form

$f_{h}^{(1)}=-\left[\sqrt{\frac{X_{B}}{2}} \int_{Y_{B}}^{X_{B}-\varepsilon} \frac{F\left(k_{1}, l_{1}, k, l, y\right)_{X=X_{B}} Y}{\left(Y^{2}-X_{B}^{2}\right) \sqrt{Y^{2}-Y_{B}^{2}}} d Y\right] C_{0} \frac{e^{i X_{B} t}}{\sqrt{t}}+O\left(\frac{1}{t}\right)$.

The term $f_{h}^{(2)}$ is estimated in exactly the same way as $f_{h}^{(1)}$ :

$f_{h}^{(2)}=-\left[\sqrt{\frac{X_{B}}{2}} \int_{X_{B}+\varepsilon}^{\infty} \frac{F\left(k_{1}, l_{1}, k, l, y\right)_{X=X_{B}} Y}{\left(Y^{2}-X_{B}^{2}\right) \sqrt{Y^{2}-Y_{B}^{2}}} d Y\right] C_{0} \frac{e^{i X_{B} t}}{\sqrt{t}}+O\left(\frac{1}{t}\right)$.

Collecting the estimates (B.25), (B.28), (B.31), (B.33), and (B.34) we obtain for $f_{h}$ :

$f_{h}=E\left(k, k_{1}\right) \frac{e^{i X_{B} t}}{\sqrt{t}}+O\left(\frac{\ln t}{t}\right)$

$E=-C_{0} \frac{e^{i X_{B} t}}{\sqrt{t}} f_{X_{B}} \frac{F\left(k_{1}, l_{1}, k, l, y\right)_{X=X_{B}} Y}{\left(Y^{2}-X_{B}^{2}\right) \sqrt{Y^{2}-Y_{B}^{2}}} d Y+i \pi C_{0} \frac{F\left(k_{1}, l_{1}, k, l, y\right)_{X=Y=X_{B}}}{2 \sqrt{2} \sqrt{X_{B}^{2}-Y_{B}^{2}}}$.

Here $f_{X_{B}}$ denotes the Cauchy principal value integral from $Y_{B}$ to infinity with the singular point $Y=Y_{B}$.

The same estimates for $f_{h}$ are obtained for the case $X_{B} \leq Y_{B}$ therefore the resulting estimate for $\tilde{v}_{s w}$ is

$\tilde{v}_{s w}=O\left(\frac{1}{t}\right), t \rightarrow \infty, x, y$ fixed.

We note that $\tilde{v}_{s w}$ in (B.36) decays faster than $f_{h}$ due to additional integration over $k$ of the integrand proportional to $e^{i t \sqrt{k^{2}+1}}$. In the periodic and "step" cases this additional integration is absent therefore 
$\tilde{v}_{s w}=O\left(\frac{1}{\sqrt{t}}\right), t \rightarrow \infty, x, y$ fixed.

Interaction IG wave - Kelvin wave

Representing the lowest-order Kelvin wave in the form

$K_{w}^{(0)} e^{-y}=e^{-y} \int_{-\infty}^{\infty} \hat{K}_{w}(k) e^{i k(x-t)} d k$

one can write the forcing term $\Phi_{0}^{(k)} \Phi_{3}^{(i g)}$ in (5.14) as follows

$\Phi_{0}^{(k)} \Phi_{3}^{(i g)}=\int_{-\infty}^{\infty} A_{1}\left(k_{1}, l_{1}\right) \hat{K}_{w}\left(k_{2}\right) e^{i\left[\left(k_{1}+k_{2}\right) x+\left(l_{1}+i\right) y+\left( \pm \omega_{k l_{1}}-k_{2}\right) t\right]} d k_{12} d l_{1}$.

Then the corresponding response is

$\tilde{v}_{k w}=-e^{-y} \int_{-\infty}^{\infty} d k_{12} \hat{K}_{w}\left(k_{2}\right) e^{\left.i\left[\left(k_{1}+k_{2}\right) x-k_{2}\right) t\right]} \int_{0}^{\infty} d l_{1} F\left(k_{1}, k_{2}, l_{1}, y\right) e^{ \pm i \omega_{k l 1} t}$,

where

$$
\begin{gathered}
F=\frac{A\left(k_{1}, l_{1}\right) e^{i l_{1} y}}{D\left(k_{1}, k_{2}, l_{1}\right)}+\frac{A\left(k_{1},-l_{1}\right) e^{-i l_{1} y}}{D\left(k_{1}, k_{2},-l_{1}\right)}=O\left(l_{1}^{2}\right), l_{1} \rightarrow 0, \\
D=\left(k_{1}+k_{2}\right)^{2}+l_{1}^{2}+2 i l_{1}-\left(\omega_{k_{1} l_{1}} \pm k_{2}\right)^{2} .
\end{gathered}
$$

Resonances are possible if $D=0$, i.e. $l_{1}=0$, but this point is also not dangerous because of (B.41). It can be shown by integration by parts that

$\tilde{v}_{k w}=O\left(\frac{1}{t^{3 / 2}}\right), t \rightarrow \infty$.

We note that really $\tilde{v}_{k w}$ decays much faster than in (B.43) since the estimate (B.43) does not take into account the integration over $k_{2}$ in (B.40).

\section{Interaction slow motion - Kelvin wave}

In the localised case the response $\tilde{v}_{k s}$ is given by formula (B.40) in which $\omega_{k_{1} l_{1}}$ is replaced by zero and $D$ takes the form

$D=k_{2}^{2}+2 k_{1} k_{2}+l_{1}^{2}+2 i l_{1}$.

Integrating by parts over $k_{2}$ one can show that

$\tilde{v}_{k s}=O\left(\frac{1}{t^{\infty}}\right), t \rightarrow \infty$. 
The estimate (B.45) is physically reasonable since the localised rapidly propagating Kelvin wave cannot efficiently interact with a localised slow motion.

In the "step" case we have the estimate

$\tilde{v}_{k s}=O\left(\frac{1}{t}\right), t \rightarrow \infty$

and in the periodic case the response does not decay in time,

$\tilde{v}_{k s}=O(1), t \rightarrow \infty$

but always

$\left\langle\tilde{v}_{k s}\right\rangle_{t}=0$

which means that the interaction is not resonant.

\section{Captions}

Fig. 1. Schematic representation of geostrophic adjustment in a half-plane bounded by a rigid wall at $y=0$.

Fig.2. Schematic representation of the lowest-order initial Kelvin wave profile $K_{w}^{(0)}(x)$ for various initial conditions. 


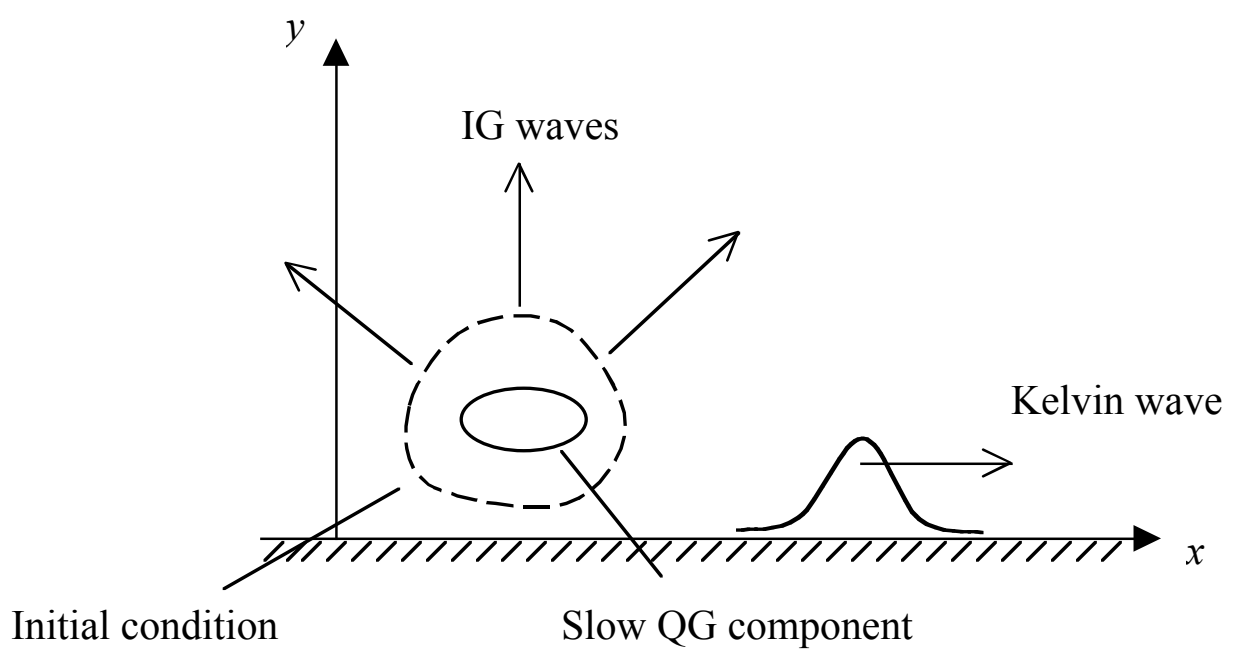

Fig. 1 


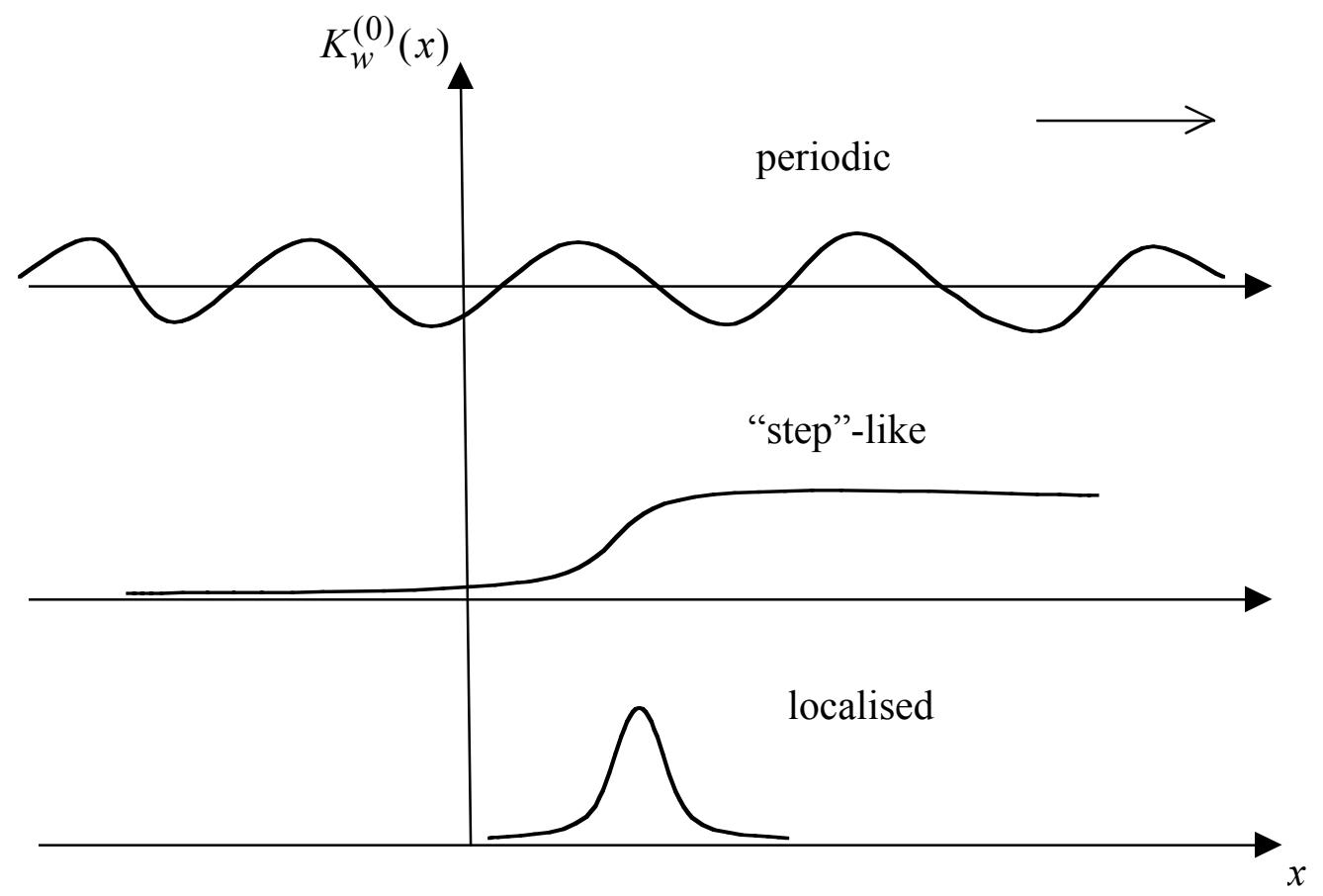

Fig.2. 